\title{
Gradhiva
}

GRADHIV

Revue d'anthropologie et d'histoire des arts

$27 \mid 2018$

Sur le vif. Photographie et anthropologie

\section{Un savoir incertain. La photographie et le document anthropologique au tournant $\mathrm{du}$ $\mathrm{XX}^{\mathrm{e}}$ siècle}

Uncertain Knowledge: Photography and the Turn-of-the-Century

Anthropological Document

\section{Elizabeth Edwards}

Traducteur : Camille Joseph

\section{OpenEdition}

\section{Journals}

Édition électronique

URL : http://journals.openedition.org/gradhiva/3503

DOI : $10.4000 /$ gradhiva.3503

ISSN : 1760-849X

Éditeur

Musée du quai Branly Jacques Chirac

Édition imprimée

Date de publication : 23 mai 2018

Pagination : $30-57$

ISBN : 978-2-35744-110-1

ISSN : 0764-8928

Référence électronique

Elizabeth Edwards, « Un savoir incertain. La photographie et le document anthropologique au tournant du xxe siècle », Gradhiva [En ligne], 27 | 2018, mis en ligne le 23 mai 2020, consulté le 07 janvier 2021. URL : http://journals.openedition.org/gradhiva/3503; DOI : https://doi.org/10.4000/gradhiva.3503 
$1 t=5 x=0$

$14=85$

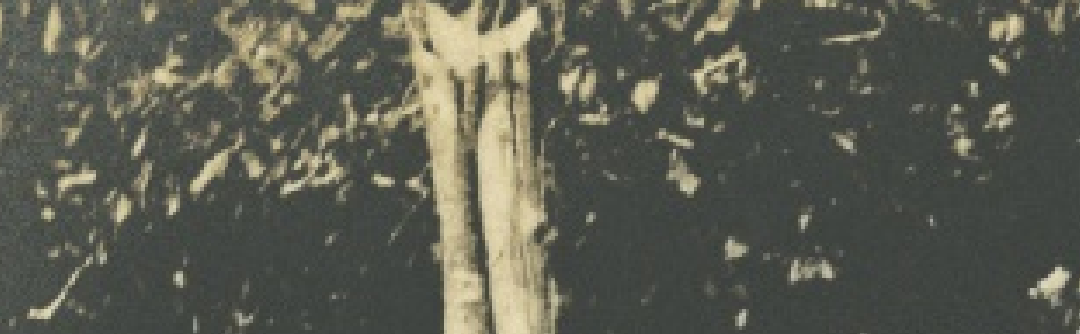

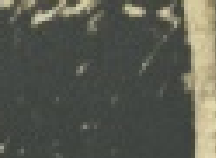

18

$3 .$.

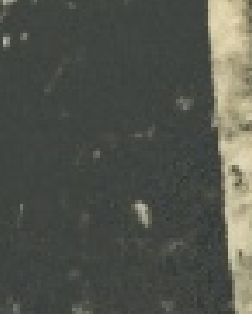

$-5$

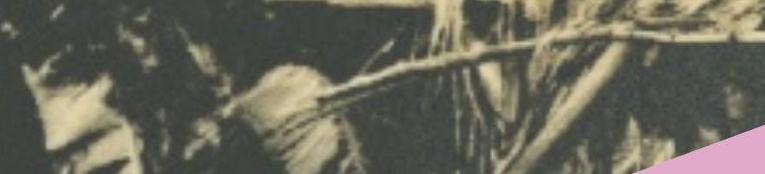

$12)^{2}=$

hity
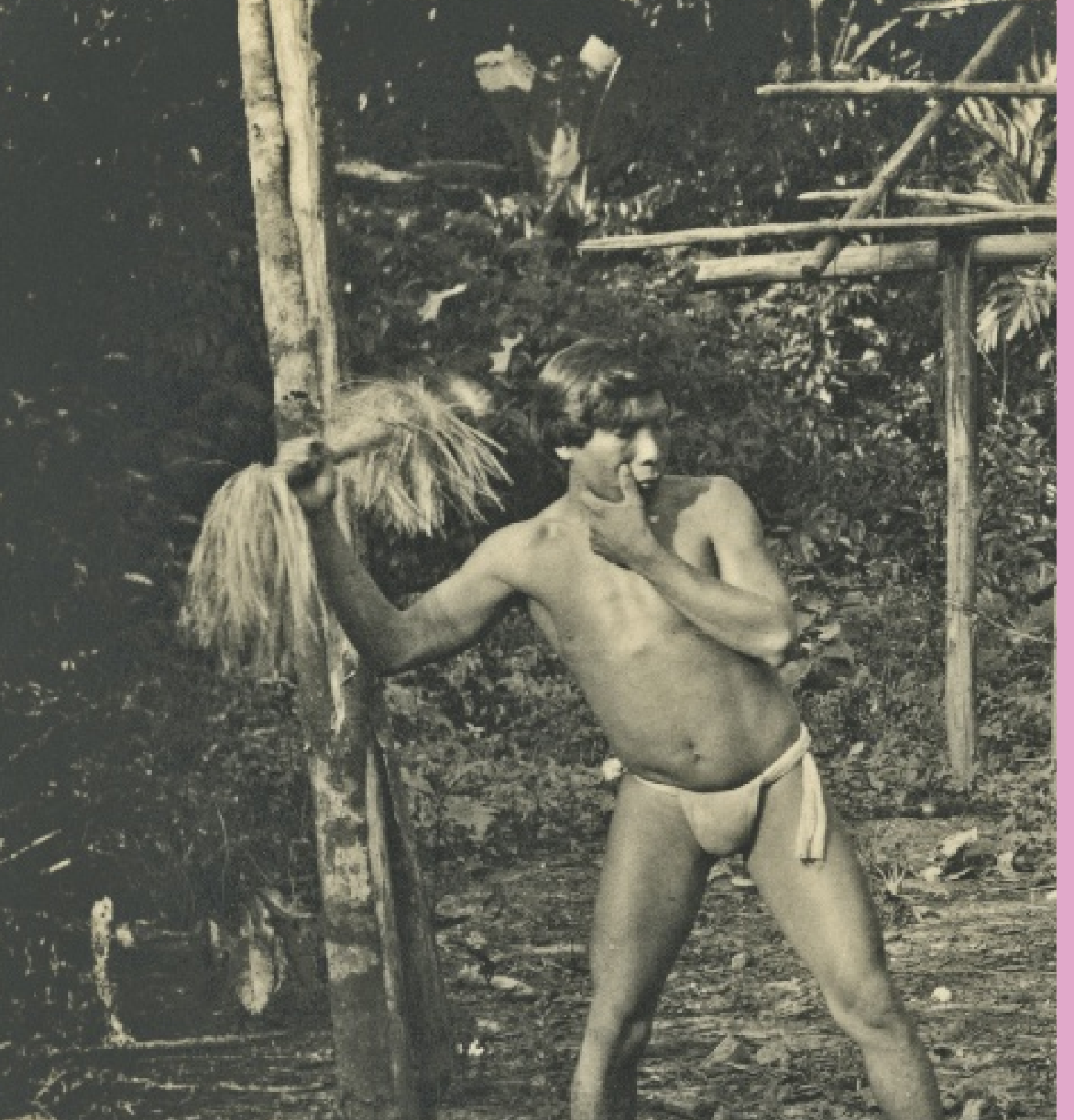

$\frac{1}{8}$
0
0
0

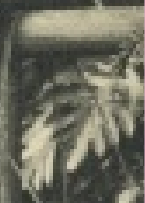

$1007 \cos ^{2}$

Cos

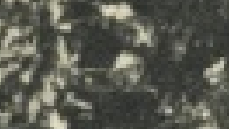

?

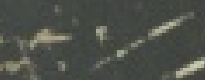

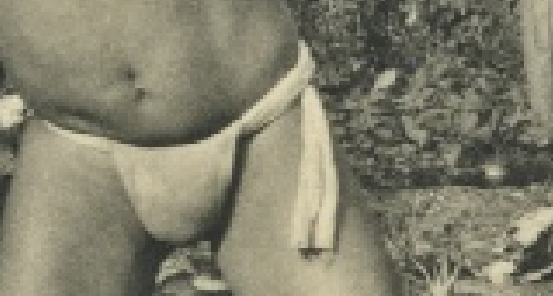

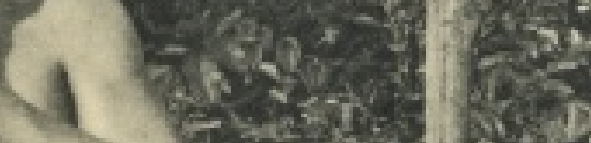

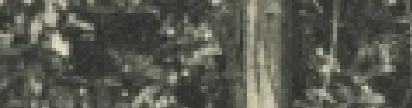
Nite cosys
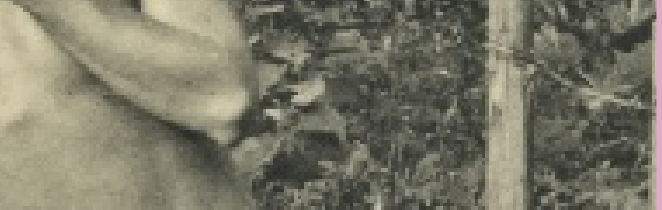

20

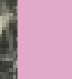

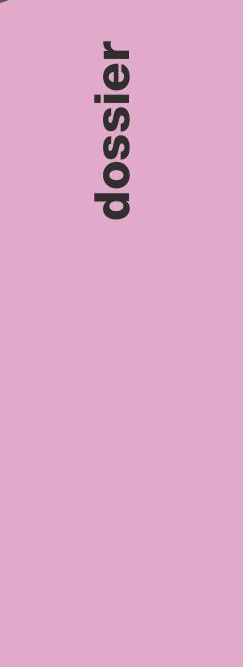


Un savoir incertain

La photographie et le document anthropologique au tournant du $x x^{\ominus}$ siècle

par Elizabeth Edwards

Cet article met en relation la production de documents photographiques en anthropologie et les changements épistémologiques qui affectèrent la jeune discipline moderne dans les années 1890. L'analyse se concentre sur trois textes rédigés à cette époque par des anthropologues britanniques, qui mettent en regard la photographie et la preuve anthropologique: celui d'Everard im Thurn en 1893, de Maurice V. Portman en 1896 et d'Alfred C. Haddon en 1899. Cette série d'instructions photographiques révèle des approches différentes et concurrentes des concepts de pertinence, de validité et d'efficacité du document visuel. Je montre que les débats qui les traversent traduisent le passage d'une objectivité mécanique à une objectivité complexe. Cela se produit notamment à travers une nouvelle appréhension de la photographie, qui ne sert plus à contrôler l'excès d'informations, mais à développer une nouvelle objectivité disciplinaire visant à saisir et analyser l'abondance de la culture elle-même.

mots clés

photographie, anthropologie britannique,

histoire, méthodologie, historiographie 
1. Le présent texte est la traduction d'un article paru sous le titre «Uncertain Knowledge: Photography and the Turn-of-the-Century Anthropological Document » (in Gregg Mitman et Kelley Wilder [dir.], Documenting the World: Film, Photography and the Scientific Record, Chicago, University of Chicago Press, 2016). Certains passages ne traitant pas directement de la thématique du numéro ont été coupés notamment sur le cinéma. [NdT]
Plutôt que d'être "lue» comme un type spécifique de littérature, l'anthropologie peut être "vue» comme un projet d'imagination visuelle 1 . Cet article examine la manière dont l'anthropologie s'est donnée à «voir » au moment même où, comme d'autres sciences, elle développait «une nouvelle vision d'elle-même" (Grimshaw 2001: 9; Pinney 2011: 15). On peut ainsi envisager la production et l'utilisation de documents photographiques comme une réponse aux bouleversements épistémologiques de l'anthropologie des années 1890 et du début du Xx $x^{e}$ siècle. À la fin du XIXe siècle furent en effet publiées toute une série de "propositions méthodologiques pour la collecte systématique de données de terrain " (Urry 1972: 45), signe du changement radical d'attitude des anthropologues de l'époque vis-à-vis de ces données et du document, qui exigeait une nouvelle approche quantitative et qualitative du concept même de preuve [evidence]. Matthew Engelke estime ainsi qu'" on peut lire l'histoire de la pensée anthropologique comme une série de débats autour de la question de la preuve" (Engelke 2008: S2-S21). II existe une littérature importante sur la posture méthodologique et théorique de l'anthropologie, mais la nature du document anthropologique et de la «preuve " elle-même a été peu étudiée, malgré le fait que son autorité soit souvent invoquée par les chercheurs en question (ibid. : S1). J'explore ici la place de la photographie dans la fabrication de documents scientifiques à un moment où l'anthropologie et ses pratiques visuelles ont évolué de manière décisive.

J'analyserai quatre textes courts mais fondamentaux, qui abordent tous la question de la production de documents photographiques dans l'anthropologie britannique du tournant du $\mathrm{xx}^{\mathrm{e}}$ siècle: un premier d'Everard im Thurn (1852-1932) de 1893, un autre de Maurice V. Portman (1860-1932) de 1896, et deux textes d'Alfred C. Haddon (1855-1940) rédigés en 1899 (avec John G. Garson) et 1912 (édité avec John L. Myres). Tous ces textes s'interrogent sur l'enregistrement efficace et démonstratif de la preuve, c'està-dire sur la façon dont la photographie devient document. Ces quatre séries d'instructions photographiques reflètent des approches différentes, parfois concurrentes, des concepts de pertinence, de validité et d'efficacité du document visuel à une époque où s'élabore une certaine tradition de l'enquête de terrain. [...]

\section{De l'excès à l'abondance}

Les instructions photographiques dont il est question ici sont en un sens des réactions aux transformations épistémologiques qui affectent l'anthropologie et réaffirment la valeur de preuve attachée à la photographie au $\mathrm{XIX}^{\mathrm{e}}$ siècle. Ces textes sont travaillés par la question de l'appréhension de la perte: comment, face à l'hybridité et à la disparition des races et des cultures, enregistrer et préserver ces dernières pour l'avenir? Ils suggèrent tous, plus ou moins implicitement, que ce sentiment de la disparition est l'une des raisons fondamentales à la fabrication de documents photographiques. Ainsi, pour im Thurn, «les phases primitives de l'existence disparaissent très vite en ces temps de voyages et d'explorations incessantes, et on devrait s'accorder à dire qu'il est du devoir des voyageurs éduqués qui se rendent dans les régions les moins connues du monde de garder une trace permanente [...] des phases qu'ils peuvent y observer " (im Thurn 1893: 184). Les remarques de Haddon sur la photographie et sa capacité 
documentaire s'inscrivent, elles aussi, dans un contexte d'ethnographie de sauvetage et d'excavation culturelle: «Les indigènes sont tous en train de mourir, et [...] ils sont à tel point modifiés par le contact [...] qu'il est de notre devoir de collecter caractéristiques physiques, artisanats, psychologie, rites cérémoniels et croyances religieuses des peuples en voie de disparition. " (Haddon 1897) On retrouve cette question de la "sauvegarde» dans la manière dont les instructions photographiques évoquent la création, le rôle et l'efficacité des documents visuels dans l'anthropologie de la fin $\mathrm{du} \mathrm{XIX}^{\mathrm{e}}$ siècle. Cette approche change néanmoins radicalement à mesure que l'anthropologie se met à prendre pour objet non plus le passé des sociétés humaines, mais leur présent.

Rédigés à l'aube d'un bouleversement épistémologique, les textes analysés ici ne présentent aucune progression chronologique ou méthodologique évidente. Au contraire, ils se chevauchent et se croisent, qu'il s'agisse des dates de leur rédaction ou des points traités, illustrant par là la difficulté à créer des documents scientifiques pertinents dans une période marquée par une relative incertitude à l'égard de nouvelles méthodes alors en plein développement. Les auteurs eux-mêmes occupent un nouvel espace au sein d'un mouvement intellectuel qui prend ses distances avec une approche scientifique des origines et des cultures humaines. Ils forment une nouvelle classe de savants, dont la première génération, incarnée par Haddon notamment, a été formée aux sciences naturelles mais se définit avant tout comme celle des «anthropologistes $\mathbf{2}$ ». Haddon lui-même observait cette évolution dans un court ouvrage intitulé History of Anthropology, dans lequel il évoquait la nature de la preuve et le rôle de la photographie:

Si les traits structurels de l'homme ont été étudiés par des hommes de formation scientifique, l'histoire de l'homme d'un point de vue culturel a surtout fait l'objet d'enquêtes réalisées par des hommes de lettres [...]. Par manque d'expérience du terrain ou en raison de leur penchant naturel à s'appuyer sur des preuves documentaires sans avoir un regard suffisamment critique sur leurs auteurs, [ils] risquent d'égarer le lecteur non averti. (Haddon 1910: 153)

Le passage d'une approche spécifiquement biologique, fondée sur des spécimens visibles, à une perspective s'efforçant de prendre en compte le contexte plus large de la culture matérielle et immatérielle peut se comprendre comme la marque d'une réorganisation générale des objets de la science. Cela est particulièrement remarquable dans le cas des sciences de terrain, où émergent de nouveaux courants méthodologiques. L'écologie, par exemple, développe alors une approche holistique de l'enquête de terrain et se penche sur les systèmes d'interrelations, ce qui n'est pas sans évoquer l'intérêt de l'anthropologie pour l'explication de systèmes culturels entiers (Alvarez Roldàn 1992). Ces perspectives plus globales se caractérisent toutes par le passage d'un contrôle de l'excès ${ }^{3}$ [excess] à une prise en compte du potentiel scientifique du désordre [messiness] de la vie quotidienne, ce que j'appellerai ici l'abondance ${ }^{4}$ [abundance]. Je montrerai que le type de documents photographiques produit dans ce contexte a joué un rôle central dans la manière dont l'anthropologie a reformulé son identité.
2. L'anthropologie du milieu du XIX ${ }^{e}$ siècle regroupait des domaines d'étude très divers: le folklore, l'archéologie, l'anatomie, la médecine, la biologie, l'étymologie et l'histoire des religions. Voir notamment Stocking 1995 ; Kuklick 1991.

Pour la période précédente, voir Sera-Shriar 2013. Arturo Alvarez Roldàn a montré comment Alfred C. Haddon avait importé en anthropologie une méthode biologique (Alvarez Roldàn 1992).

3. J'emprunte le terme à Deborah Poole (2005), qui a livré une analyse très importante de l'" excès" et de son rapport avec l'anthropologie, la race et la photographie.

4. Quelques précisions sur l'analyse de «l'excès » chez Poole: selon elle, la fin du XIX ${ }^{e}$ siècle est marquée par la nécessité, pour les anthropologues, de ne pas se laisser submerger par le flot d'informations que la technique photographique n'était pas apte à «trier ».

Ils cherchèrent ainsi à maîtriser ce qui apparaissait sur les images, à isoler l'information à traiter, en éliminant notamment les éléments de contexte superflus pour leurs analyses, au moment de la prise de vue ou a posteriori. Dans le cas des photographies de types raciaux en particulier, les anthropologues élaborèrent des méthodes de standardisation

- longueurs de focale, poses, arrière-plans, détourages des corps qui devaient permettre de supprimer les traces visibles de la culture du sujet photographié (et les expressions du visage), et de constituer une documentation homogène [NdT]. 
5. Cet article, accompagné de planches comportant des épreuves sur papier albuminé, est le premier texte anthropologique illustré (Lamprey 1869).

6. Dans la lutte pour la reconnaissance disciplinaire, les études folkloriques [folk studies] britanniques, qui étaient de plus en plus perçues comme descriptives et anciennes, furent marginalisées. Elles ne parvinrent jamais à obtenir la respectabilité, le statut et l'importance que cette discipline acquit aux États-Unis et dans d'autres pays européens.

7. Voir par exemple Tucker 2005

8. Pour une étude du rôle de la photographie dans le développement de cette institution influente pour l'anthropologie du xIx ${ }^{\mathrm{e}}$ siècle, voir Poignant 1992.

\section{Contrôler le document photographique}

Les photographies circulaient dans tous les réseaux de l'anthropologie de la seconde moitié du XIX siècle, qui s'en servait notamment pour identifier les races, les classer et étudier la culture matérielle. Cependant, l'attention portée à la qualité et à la quantité des images montre que, de plus en plus, leur validité scientifique est fixée par des critères et des méthodes d'observation propres à la discipline (Pinney 2011: 17-62). Des images d'origines très différentes, commerciales et scientifiques, étaient en effet utilisées par les anthropologues. Mais plus que leur intention ou leur style formel, c'est le sujet de ces photographies et leurs modes de consommation qui en faisaient des documents scientifiques, ainsi qu'en témoigne la grande diversité de formats et de styles dont on jugeait alors qu'ils présentaient «un intérêt anthropologique» (Edwards 2001: 27-50; Pinney 2003: 3). Dans le domaine de l'anthropométrie en particulier, on tenta de systématiser l'usage de la photographie en développant notamment une cartographie somatique [somatic mapping] des corps et donc des races. Ces pratiques visuelles visaient avant tout à créer des documents mathématiquement lisibles où l'excès se trouvait rigoureusement contrôlé et l'image ontologiquement «purifiée » (Pinney 2013: 34). La méthode la plus célèbre est celle de John Lamprey, qui avait élaboré un système dans lequel l'individu photographié était placé devant une grille quadrillée (fig. 1). Le procédé, décrit dans "On a Method of Measuring the Human Form» et illustré par des photographies de Henry Evans, fut publié dans le Journal of the Ethnological Society en $1869^{5}$.

L'année suivante, peut-être en réponse à la méthode de Lamprey, Thomas Henry Huxley élabora un système encore plus rigoureux de photographie anthropométrique, qui circula dans les colonies britanniques (Edwards 2001: 131-155). Ces études montrent déjà un intérêt pour des questions d'ordre culturel, la culture, selon une approche hiérarchique et évolutionniste des races, étant alors considérée comme biologiquement déterminée. Mais les pratiques tant anthropologiques que photographiques de la fin $d u$ XIX ${ }^{e}$ siècle révèlent surtout un intérêt grandissant pour la production de documents visuels prenant pour objet les pratiques culturelles ellesmêmes. II ne s'agissait pas d'une simple collecte de faits illustrant des idées générales: ces documents étaient le résultat d'une étude approfondie des cultures observées (Stocking 1995 : 95).

La nouvelle perception que les «anthropologistes » développèrent d'eux-mêmes et la professionnalisation du savoir anthropologique, en particulier vis-à-vis d'autres sciences comme l'anatomie, la géographie et les études folkloriques ${ }^{6}$, sont intimement liées à l'apparition de certains types de documents. Le savoir anthropologique du XIX siècle reposait sur un modèle de collecte de données hérité $d u X \mathrm{VIII}^{\mathrm{e}}$ et du début du XIX ${ }^{\mathrm{e}}$ siècle, selon lequel les observateurs et collectionneurs amateurs rapportaient de la périphérie le matériau destiné à être interprété dans les métropoles - processus que l'on désigne aujourd'hui de manière quelque peu péjorative par l'expression «anthropologie de cabinet» [armchair anthropology ${ }^{7}$ ]. L'Institut d'anthropologie britannique [Anthropological Institute], principal lieu d'échange entre amateurs et anthropologues professionnels, avait pour mission d'encourager la production de documents à travers la collecte de données ethnographiques ${ }^{8}$. II est remarquable que les instructions dont il est question ici furent, 


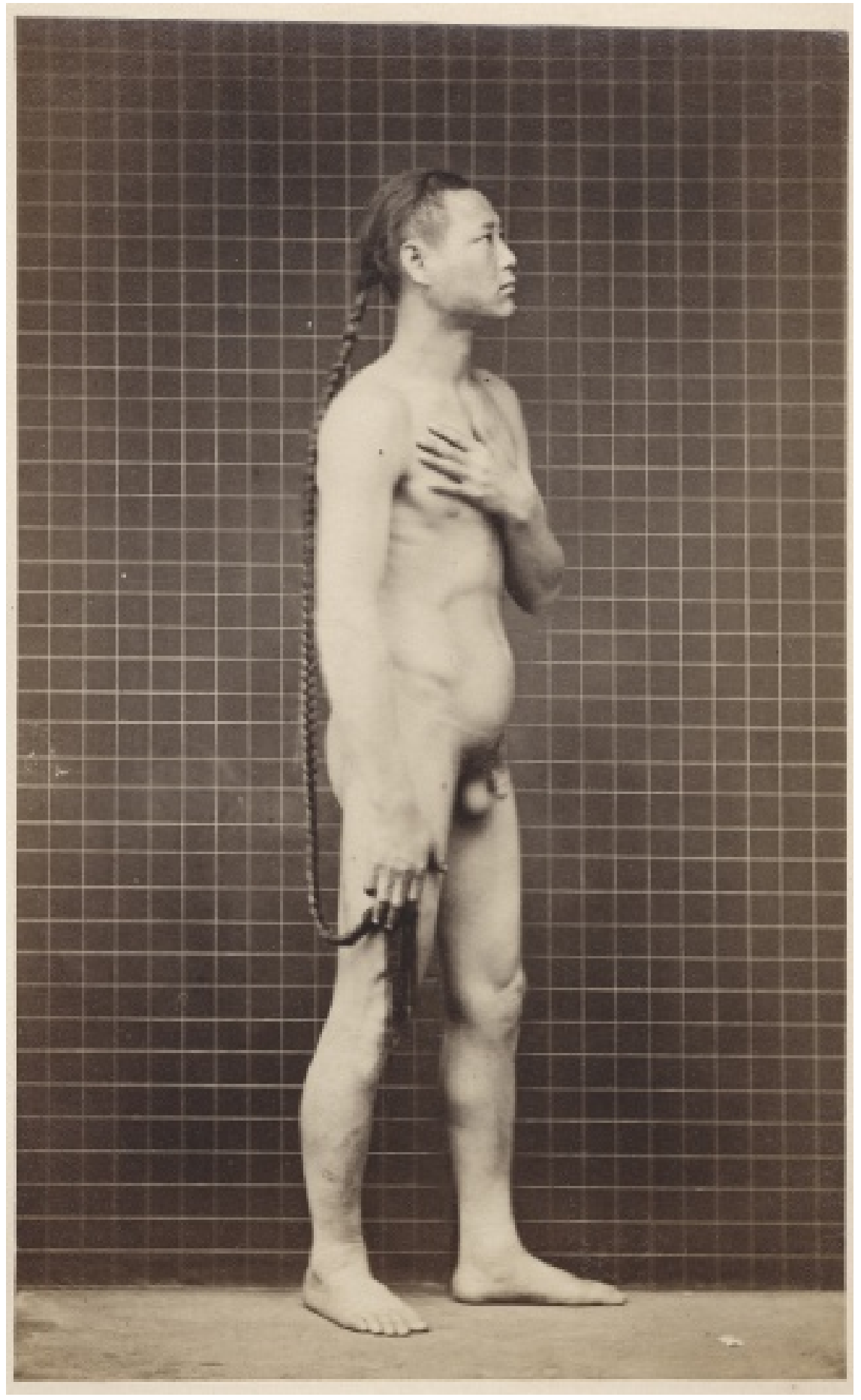

fig. 1

Henry Evans, Photographie anthropométrique utilisant la grille orthonormée du système de John Lamprey, 1869, épreuve sur papier albuminé. Avec l'aimable autorisation du Pitt Rivers Museum, University of Oxford, 1945.5.97.3. 
9. Pour une étude détaillée de l'origine, de l'évolution et de l'impact des quatre éditions des N \& Q publiées entre 1870 et 1920 , et pour une comparaison avec des questionnaires plus anciens, voir Urry 1972. II existe également une série de questionnaires datant du début du XIX ${ }^{e}$ siècle qui anticipent à certains égards les $N \& Q$ sur la question de la gestion de l'observation (Sera-Shriar 2013: 53-79). On trouve des instructions et des questionnaires semblables ailleurs, notamment dans l'anthropologie française et allemande, où se reflètent les préoccupations méthodologiques propres de ces disciplines. Voir à ce sujet Dias 1994 . Neumayer 1875.

10. $N \& Q, 1874$ : iv. C'est l'auteur qui souligne. d'une manière ou d'une autre, toutes produites sous ses auspices dans la dernière décennie du XIX ${ }^{\mathrm{e}}$ siècle. À partir de 1874, I'Institut publia un petit ouvrage en association avec l'Association britannique pour l'avancement de la science [British Association for the Advancement of Science], les Notes and Queries on Anthropology (désormais $N \& Q$ ). Dans ce volume destiné aux amateurs travaillant sur le terrain, notamment les missionnaires, les officiers coloniaux et tout savant intéressé par des questions anthropologiques, la production de savoir prenait la forme d'un questionnaire organisé par domaine de collecte: croyances religieuses, danses, armes, manière de saluer, etc ${ }^{9}$. Le texte fournissait non seulement des conseils sur les éléments à documenter, mais aussi, comme le révèle la structure même de l'ouvrage, des explications sur la manière «objective» de procéder. Les $N \& Q$ eurent une influence décisive, directement ou indirectement, sur les autres instructions relatives à la photographie, qui trouvèrent là une inspiration et une perspective nouvelle pour définir le type de documents visuels produit par l'anthropologie naissante.

Si l'efficacité documentaire de la photographie allait de soi, dans la pratique, la production de documents lisibles s'avérait problématique. L'objectivité mécanique de l'appareil photographique et sa capacité à enregistrer la réalité n'étaient pas remises en question. Mais les textes que j'examine ici s'inquiétaient, comme d'autres sciences à la même époque, de la «bonne exécution » permettant d'aboutir à des documents empiriquement fiables, compréhensibles et pouvant servir de preuves (Daston et Galison 1992: 82). Ainsi, le document ne dépendait pas uniquement de l'appareil photographique, mais également de la manière dont l'objet, celui de la photographie comme celui de la discipline, était présenté à l'appareil. Cette question devint particulièrement urgente à mesure que, dans un contexte d'expansion de l'administration coloniale et de développement des techniques photographiques, la diversité et la quantité de matériaux ne cessaient d'augmenter. Cela explique peut-être la somme de textes consacrés à la photographie anthropologique parus dans les années 1890, moment où la nature du document allait connaître une évolution décisive, sous l'influence de la photographie et du cinéma, mais également des modes de collecte, d'évaluation et d'archivage caractéristiques du XIX siècle d'un côté, et des méthodes de l'anthropologie protomoderne de l'autre. [...]

La nécessité de plus en plus urgente de définir une «bonne exécution » se manifeste dans l'attention que les éditions successives des $N \& Q$ ont accordée à la photographie et, par suite, aux documents visuels. II faut attendre la troisième édition (1899) pour voir apparaître un long passage consacré à la photographie, celle-ci étant seulement citée auparavant parmi les différents outils méthodologiques. La première édition (1874) comporte bien une rubrique «Photographie», mais elle est vide. On a expliqué cela par un problème que les éditeurs connaissent bien: le contributeur n'aurait pas rendu son texte. Mais j'avancerais une autre hypothèse: on peut voir là l'idée selon laquelle l'image se suffit à elle-même. En effet, cette omission était justifiée par une référence à la qualité des documents produits: "Plutôt que de placer [les sections manquantes] entre des mains moins expertes, elles ont pour l'instant été omises et seuls les titres ont été insérés ${ }^{10}$. 》 La deuxième édition (1892), dirigée par la même équipe, comporte à peine deux pages et demie de conseils techniques. La troisième (1899) reprend presque à l'identique la précédente, mais la rubrique «Photographie» y est sensiblement augmentée, avec près de onze pages. 
Cette troisième édition des $N \& Q$, qui paraissaient pourtant depuis bientôt trente-trois ans déjà, présente ainsi la première analyse complète, à la fois technique et théorique, consacrée à la photographie. Celle-ci reste cependant fidèle à l'anthropologie du XIX ${ }^{e}$ siècle, insistant sur une description de surface et une cartographie somatique au service d'une anthropologie physique racialiste. À côté des détails techniques et des instructions anthropométriques fournis par Garson (1861-1932), spécialiste d'anthropologie physique, figure le texte rédigé par Haddon. Celui-ci y évoque d'autres formes d'anthropologie exigeant des «documents " adéquats et met en regard le potentiel de l'abondance et les dangers de l'excès. L'article de Haddon fut très peu modifié entre la troisième (1899) et la quatrième (1912) édition, alors même que le cadre intellectuel et méthodologique de la production de documents avait, lui, profondément changé: pour reprendre les termes de George Stocking, on avait désormais le sentiment d'avoir «mis un pied dans l'univers ethnographique du [xxe] siècle» (Stocking 1995: 95). [...]

\section{Everard im Thurn : photographier des "êtres vivants"}

Officier colonial posté en Guyane britannique dans les années 1880 et 1890, Everard im Thurn, botaniste de formation, fut également conservateur de musée 11. En 1893, il publia dans le Journal of the Anthropological Institute un article intitulé "Anthropological Uses of the Camera" ["Les usages anthropologiques de la photographie»], rédigé à partir d'une conférence avec des projections à la lanterne magique qu'il avait donnée à l'Institut la même année, résultat de quinze ans passés dans les colonies ${ }^{\mathbf{1 2}}$.

Im Thurn s'intéressait avant tout à « la photographie pour son enregistrement fidèle, pas simplement du corps des primitifs - qui peuvent d'ailleurs être mesurés et photographiés de manière plus précise morts que vivants, à supposer qu'il soit facile de les obtenir dans cet état - mais de ces peuples en tant qu'êtres vivants " (im Thurn 1893: 184). On a pu voir dans ce passage l'émergence d'une approche plus humaniste de la discipline anthropologique et de la production de documents scientifiques, ainsi que le signe d'un intérêt pour le «vif et le vivant» par opposition à «l'immobile et au silencieux ${ }^{\mathbf{1 3}}$ ». Mais ce texte représente également le début d'une nouvelle perspective à l'origine de l'évolution décrite plus haut: le passage d'un excès d'informations nécessitant contrôle et cadrage à une abondance de potentialités scientifiques. Cette citation quelque peu anecdotique d'im Thurn marque ainsi l'apparition du culturel dans l'image, non plus simplement saisi à partir du biologique mais désormais compris comme l'ensemble des expériences quotidiennes incluant familles, amis, maisons, gestes, langage corporel et autres éléments sociaux (fig. 2).

Im Thurn poursuit: «De la même manière que les photographies purement physiologiques des spécialistes d'anthropométrie ne sont que de simples images de corps sans vie, les photographies ordinaires d'indigènes anormalement misérables [...] peuvent être comparées aux clichés que l'on voit parfois d'animaux mal empaillés et déformés. » (im Thurn 1893: 186) Malgré l'accent mis sur l'aspect plus naturaliste qu'anthropométrique, l'approche défendue par im Thurn reposait dans le même temps sur un discours racialiste de la pureté et du métissage. On voit ici surgir une tension entre, d'un côté, les styles plus anciens de l'enregistrement anthropométrique
11. Im Thurn effectua une brillante carrière d'officier colonial, qu'il termina comme gouverneur général de Fidji (1904-1908) puis haut commissaire pour le

Pacifique occidental (1908-1911).

Voir Dalziell 2007.

12. Ces diapositives sont conservées aux National Museums of Scotland à Edimbourg. Pour une présentation très utile des conférences avec projections et de leurs discours raciaux et moraux, voir Cox 2007.

13. Voir Tayler 1992 Pour une critique de cette interprétation, voir Cox 2007 ; Pinney 1992: 78. 


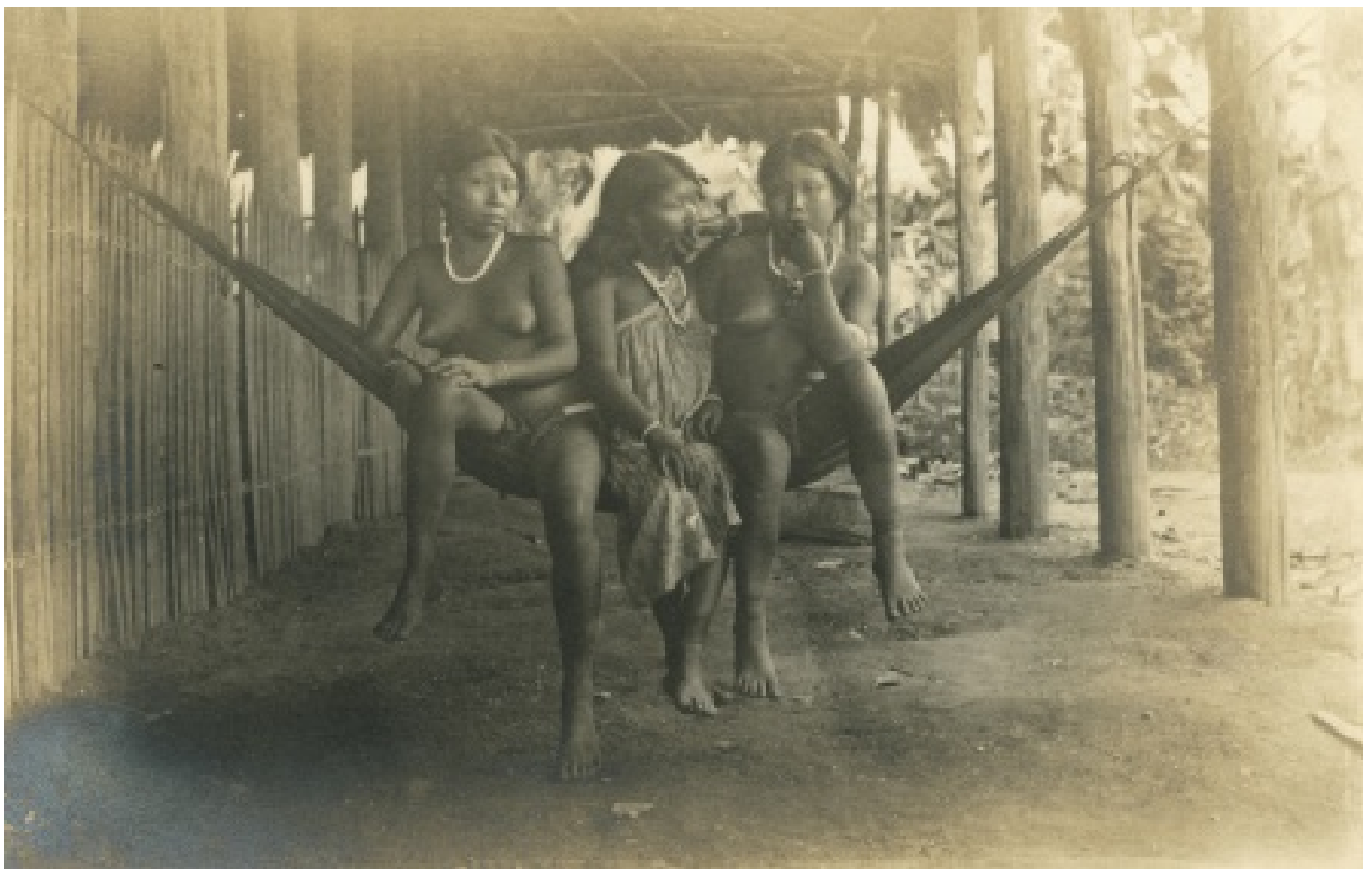

fig. 2

Everard im Thurn,

Jeunes femmes Warrau

dans un hamac, Guyane

britannique, vers

1883-1884, épreuve

sur papier albuminé.

Royal Anthropological

Institute, Londres

(c) RAl 636. 
et les paradigmes biologiques de l'anthropologie et, de l'autre, la tradition émergente de l'enquête de terrain de l'anthropologie culturelle, qui revisite la tradition tylorienne comparatiste et évolutionniste.

Les illustrations du texte d'im Thurn représentent des groupes d'individus au repos, se prenant dans les bras, flânant, jouant, etc. : bref, une saisie de l'abondance. Dans le même temps, elles reflètent des pratiques photographiques caractéristiques de la production de document photographique de la période précédente, l'arrière-plan étant souvent supprimé afin de focaliser l'attention sur le corps du sujet. Dans le portrait de Lonk, par exemple, c'est un ornement de cou spécifique qui ressort (fig. 3).

Cette pratique est à rapprocher de la neutralisation des arrière-plans dans l'atlas photographique de Carl Damman, Anthropologisch-ethnologisches Album in Photographien, publié vingt ans plus tôt. Destinées à un usage commercial, les photographies de format «carte de visite» rassemblées par la Berliner Gesellschaft für Anthropologie, Ethnologie und Urgeschichte [Société berlinoise d'anthropologie, d'ethnologie et de préhistoire] avaient été transformées en documents «anthropologiques» par un traitement consistant à éliminer tout élément de contexte à l'arrière-plan et à supprimer les traits culturels au profit des caractéristiques biologiques ${ }^{14}$. D'où la question de savoir où se situait le «naturalisme» prôné par im Thurn: dans l'image ou dans le rapport du corps de l'ethnographe au terrain? Le naturalisme des gestes et des attitudes corporelles sur lequel insiste im Thurn se fige, non seulement par l'action de l'appareil photographique, mais aussi parce que l'image doit servir de document. Car si les clichés rendent plus lisibles certaines formes culturelles, ils versent simultanément dans une tradition d'appréhension biologique de l'authenticité culturelle.

Le texte d'im Thurn sur la photographie peut être interprété comme une réaction aux possibilités nouvelles offertes par ce médium à une époque historique donnée. Mais, comme Anna Grimshaw l'a remarqué, les stratégies des anthropologues reposent sur des méthodes d'observation spécifiques qui présupposent des relations particulières avec le monde, affectant en retour la nature même du document produit (Grimshaw 2001: x-xi). Im Thurn était bien conscient de l'oscillation permanente entre objectivité et subjectivité dans la création de documents pour la jeune anthropologie culturelle, fondée sur le désordre de l'expérience. La photographie d'«êtres vivants» plutôt que de spécimens statiques (fig. 4) était «une opération extrêmement complexe, plus rarement pratiquée par les anthropologues, et dont j'imagine que l'utilité pour l'anthropologie [...] en tant que science exacte sera dans un premier temps mise en doute par certains» (im Thurn 1893: 184). Im Thurn faisait de nouveau ici référence aux tensions et aux glissements entre, d'un côté, le contrôle de l'excès et, de l'autre, la valorisation de l'abondance, qui devient dès lors signifiante à la fois du point de vue de la culture étudiée et de la discipline.

\section{"Des réponses exactes": Maurice V. Portman}

Le texte d'im Thurn est suivi trois ans plus tard par une autre série d'instructions rédigées cette fois par Maurice V. Portman, officier colonial des îles Andaman, dans la baie du Bengale. Ces îles, transformées en bagne par les Britanniques après le soulèvement de 1857, fascinaient depuis longtemps les anthropologues, qui pensaient que leurs habitants, les « Négritos », étaient longtemps restés dans un état d'isolement exceptionnel (Edwards 1992;
14. De fait, l'album de Dammann contient des "cartes de visite" d'Arawak et de Karib, selon toute vraisemblance celles-là mêmes qui ressemblaient selon im Thurn à des photos "d'animaux mal empaillés". Voir Edwards 1990 et Poole 2005. 


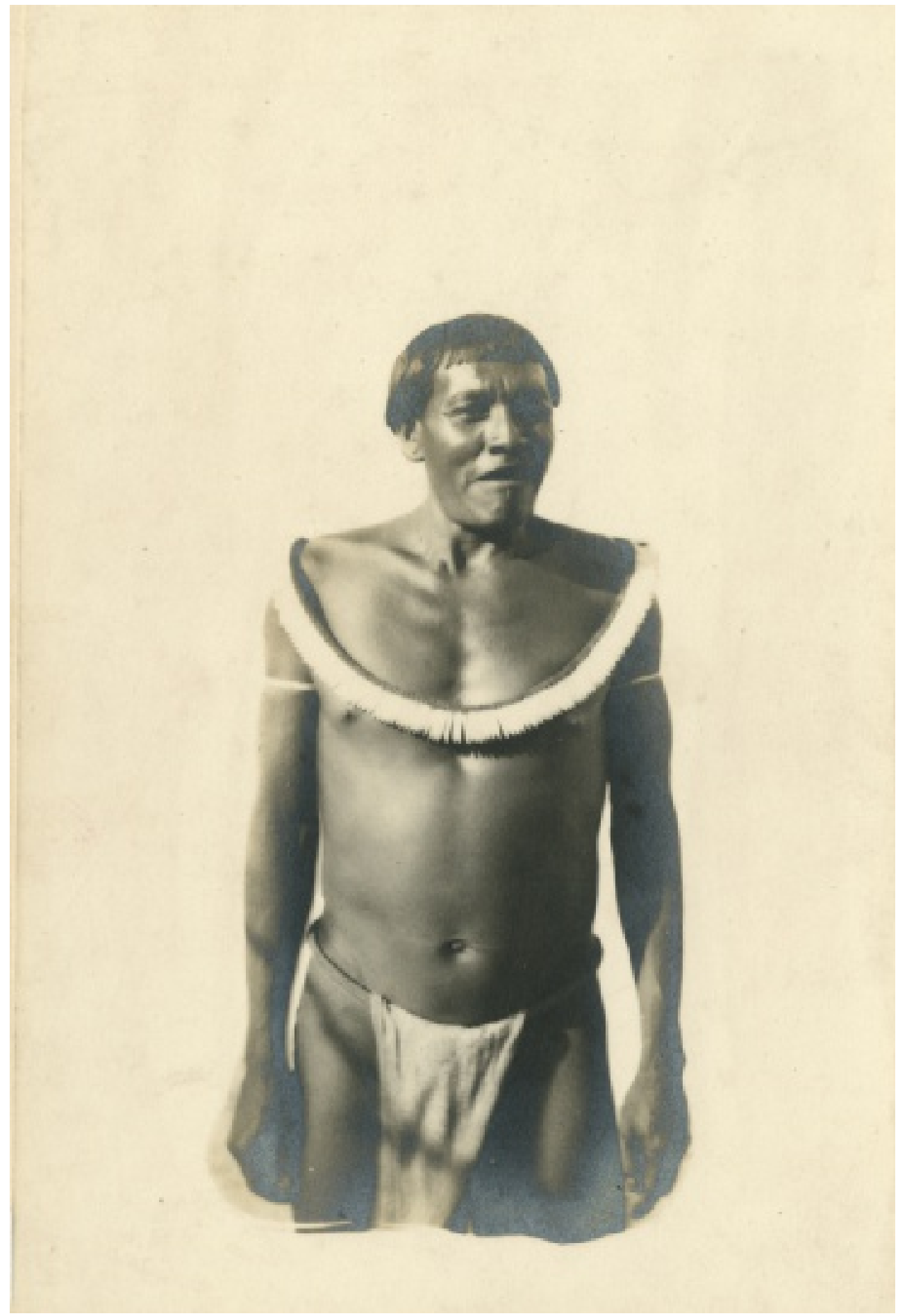

fig. 3

Everard im Thurn,

Lonk portant un large

collier de plumes,

Guyane britannique

vers 1883-1884, épreuve

sur papier albuminé. Royal

Anthropological Institute,

Londres @ RAI 618. 


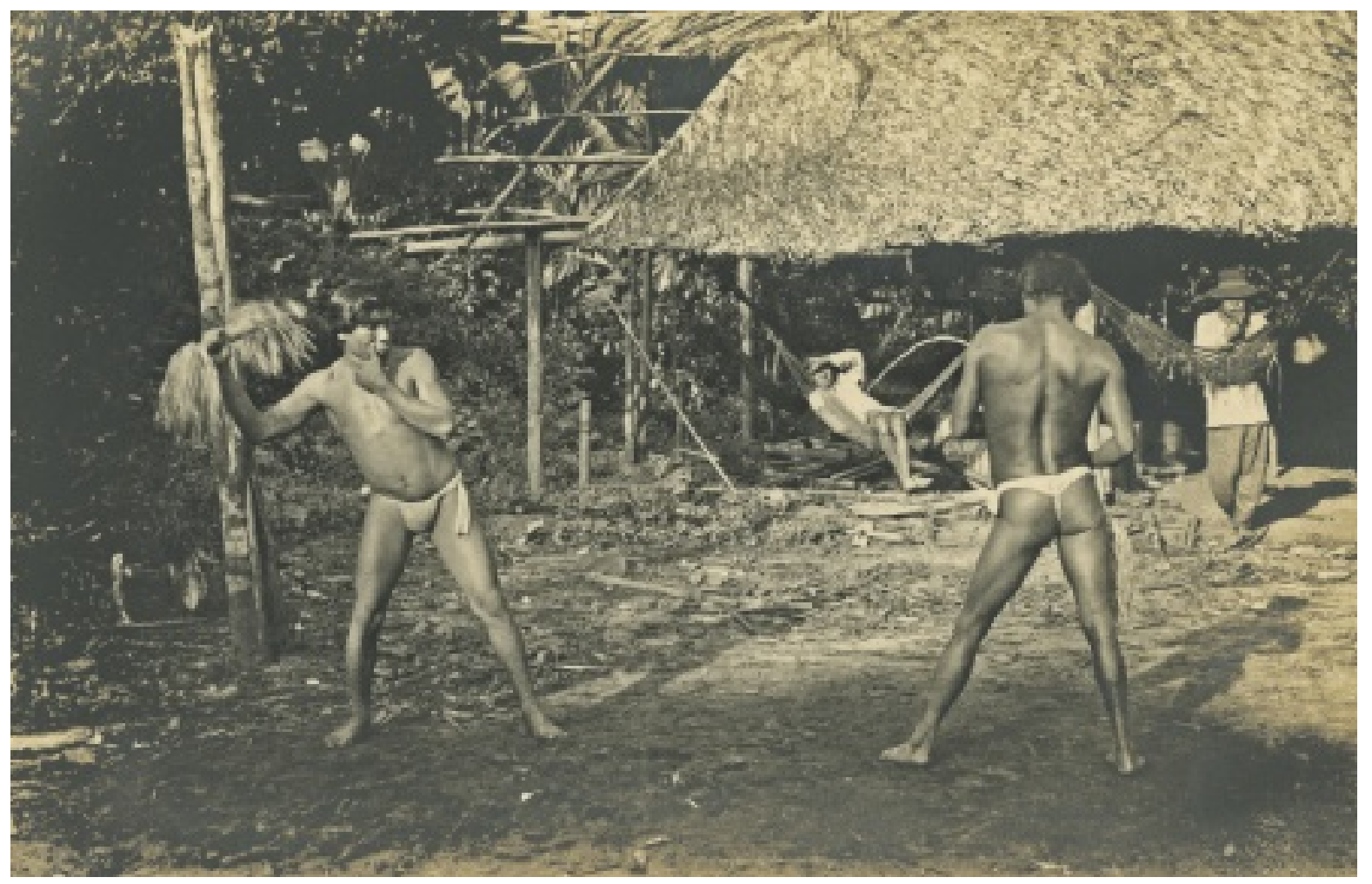

fig. 4

Everard im Thurn, Jeunes

hommes Warrau jouant,

Guyane britannique, vers

1883-1884, épreuve sur

papier albuminé. Royal

Anthropological Institute,

Londres () RAI 641. 


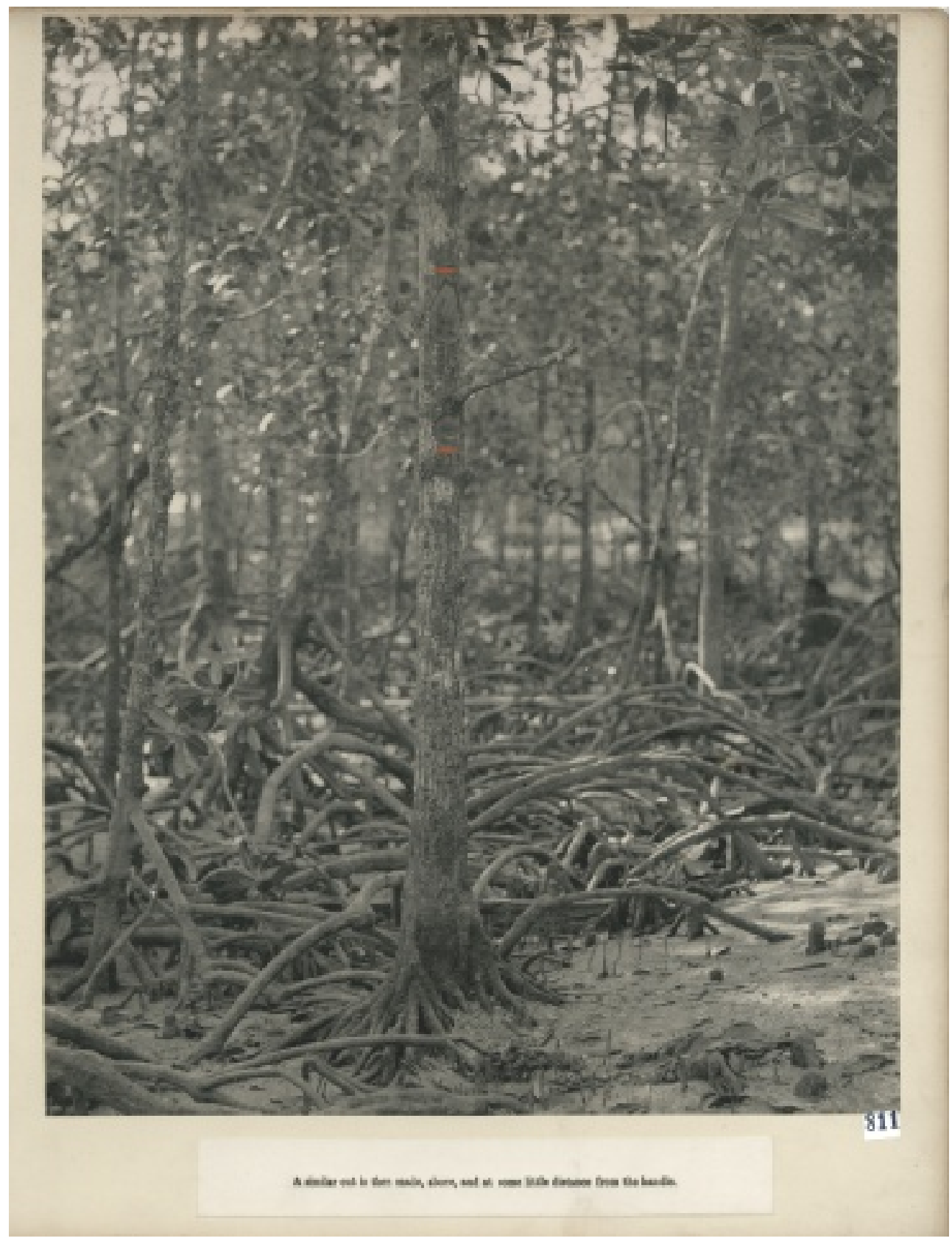

fig. 5

Maurice V. Portman,

Arbre de la mangrove

sur lequel on a indiqué la

portion qui sert à fabriquer

l'herminette. Les marques

ont été repassées au crayo

rouge, vers 1893-1894,

épreuve au platine. Royal

Anthropological Institute,

Londres () RAI 811. 
Tomas 1991). Ils furent dès lors très largement représentés dans les images et textes anthropologiques, en particulier grâce aux efforts du premier officier en charge du territoire, E. H. Man, dont l'activité quasi obsessionnelle de collecte et de photographie de la culture andamaise fournit aux institutions européennes de très nombreux documents scientifiques ${ }^{\mathbf{1 5}}$.

Les instructions rédigées par Portman et présentées à I'Institut d'anthropologie en 1895, avant d'être publiées dans la revue de l'Institut l'année suivante, sont liées à son expérience d'ethnographe colonial et aux très nombreuses photographies de la culture andamaise qu'il réalisa pour le compte du British Museum. En 1889, Portman avait proposé au musée de prendre des clichés des différentes étapes de fabrication de divers artefacts andamais. Ce projet, achevé en 1893, fut envoyé à la fois au musée et aux autorités britanniques en Inde (Pinney 1997: 68) [fig. 5]. Les clichés comprenaient également un nombre important de portraits anthropométriques d'Andamais, de face et de profil. Ils étaient accompagnés des mensurations correspondantes, comme le préconisait la première partie des $N \& Q$ consacrée à l'anthropologie physique ${ }^{\mathbf{1 6}}$. II en résulta vingt volumes d'épreuves, tirées selon un procédé au platine pour garantir leur pérennité ${ }^{17}$. [...]

Portman insistait sur la nécessité de maîtriser rigoureusement la production de documents afin d'obtenir des spécimens contrôlés et des documents viables «fournissant des réponses exactes» (Portman 1896: 76). L'excès d'informations sur les photographies de type naturaliste obscurcissait selon lui leur dimension scientifique. À l'exception de quelques clichés de terrain, indispensables pour montrer de quel arbre particulier provenait tel bois, par exemple, les photographies de Portman ont un fond neutre qui rappelle les arrière-plans effacés des photographies plus anciennes. La lumière est soigneusement travaillée, de sorte que le regard est dirigé, l'excès contrôlé et un moment précis de la pratique isolé pour l'analyse, à la manière d'un travail de laboratoire: «L'ethnologie requiert de la précision. II faut éviter un éclairage trop faible et une photographie pittoresque; il faut uniquement se soucier de la luminosité générale et vérifier qu'aucune arme ou partie du corps ne gêne en cachant des objets qu'il serait important de voir. » (Ibid.: 77) L'objectif était d'obtenir un maximum de visibilité scientifique. L'attention du spectateur était en outre orientée par des légendes courtes, expliquant par exemple la fabrication d'une herminette: «L'extrémité est ensuite coupée à la bonne longueur et polie. Le dos est de la même façon taillé et ciselé de près en une ligne recourbée semblable à celle de la poignée ", etc. (fig. 6 et 7).

Néanmoins, la photographie restait le document le plus important: «Des photographies bien prises, auxquelles seront ajoutées des légendes explicatives, constituent les meilleures réponses au questionnaire figurant dans les Notes and Queries. " (Ibid.: 76) La photographie devenait, pour reprendre la formule de Satadru Sen, une «forme d'interrogation, une extraction des connaissances » (Sen 2009: 371). L'appareil photographique ne permettait pas seulement de répondre au questionnaire figurant dans les $N \& Q$ par le biais de légendes claires et explicatives, il fournissait un document complet. II est important de souligner que le document était ici le produit d'une inversion consciente de la hiérarchie traditionnelle entre texte et image, l'appareil photographique étant une machine garantissant l'authenticité scientifique. [...]

Le sujet de la photographie acquiert, dans les photographies de Portman, une visibilité scientifique mise en scène à travers toute une série de médiations, ce que Christopher Pinney a appelé, à la suite de Bruno Latour,
15. On trouve notamment des photographies de Man dans les fonds des musées ethnologiques de Londres, Oxford, Vienne, Berlin et Hambourg.

16. Les photographies anthropométriques furent réalisées par William Molesworth, capitaine chirurgien au sein de l'Indian Medical Service. En 1894, Portman et Molesworth publièrent un volume de clichés et de données anthropométriques, systématiquement présentés sous forme de fiches et de grilles. Les chiffres, en particulier, participaient du système de contrôle exercé par le pouvoir colonial (Sen 2009).

17. Quelque vingt-six volumes contenant le matériau rassemblé par Portman, pour l'essentiel des photographies, sont conservés au British Museum. Une autre partie importante de la collection se trouve à la British Library. 


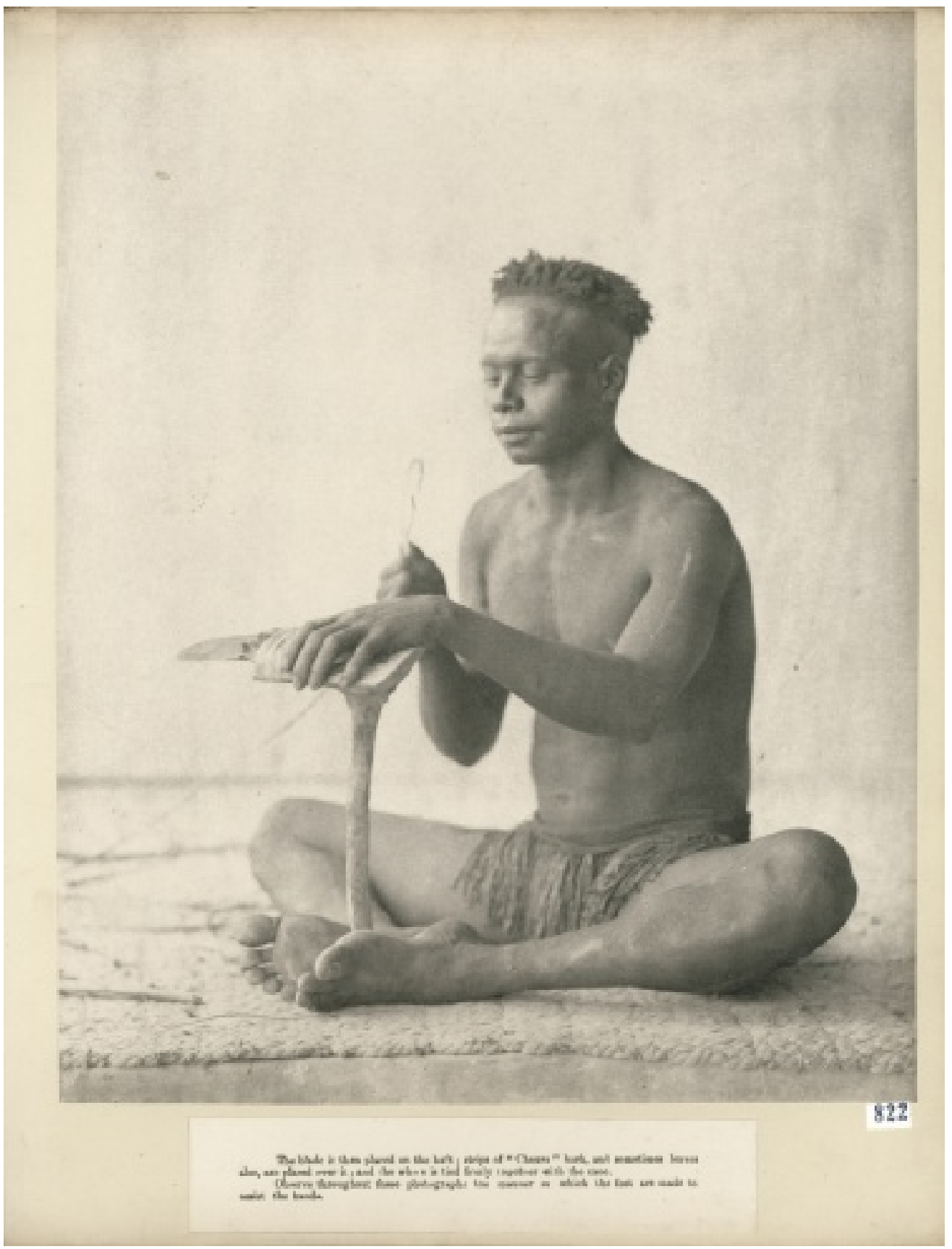

fig: 6

Maurice V. Portman,

Fabrication d'une

herminette. On a pris soin

de placer le sujet devant

un arrière-plan neutre,

vers 1893-1894,

épreuve au platine.

Royal Anthropological

Institute, Londres

(c) RAI 822. 


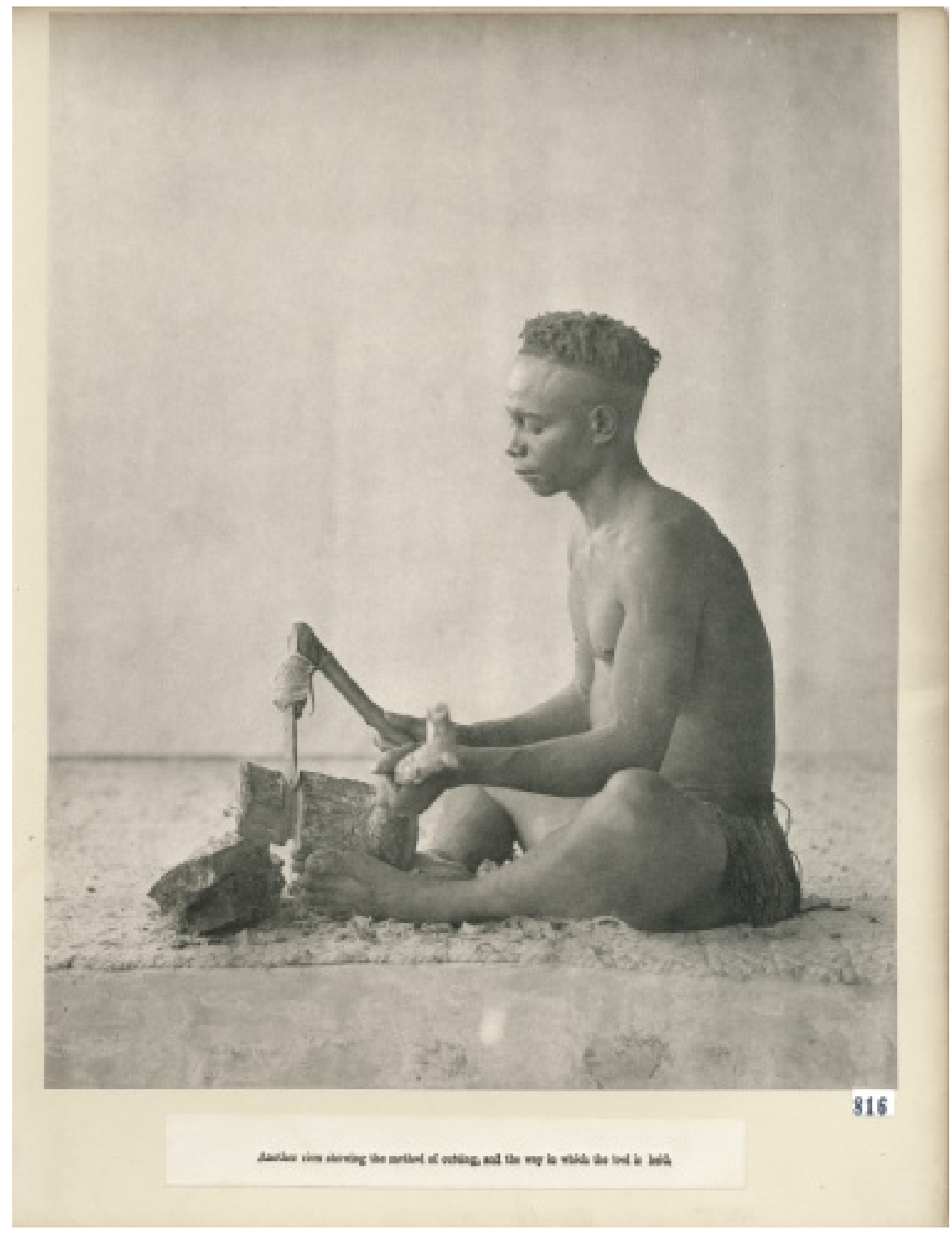

fig. 7

Maurice V. Portman,

Autre vue montrant

la manière de tailler le bois

et de tenir l'outil, vers 1893-

1894, tirage platine-

palladium cartonné.

Royal Anthropological

Institute, Londres

(c) RAI 816. 
18. Sur l'émergence de l'anthropologie moderniste et ses pratiques visuelles, voir Grimshaw 2001 : 15-31; Urry 1972.

19. Comme beaucoup d'hommes de sa génération, Haddon avait été formé aux sciences naturelles, en particulier à la zoologie et à la biologie. On lui attribue l'introduction du terme d'" enquête de terrain " [fieldwork] dans le lexique anthropologique (Stocking 1983: 27). une «purification» vers une «zone ontologique distincte», ici le document anthropologique (Pinney 2013: 34). On est loin du concept de naturalisme d'im Thurn. Produire un document dans le but de montrer scientifiquement quels gestes techniques accompagnent la fabrication d'une herminette, par exemple, nécessitait de placer le sujet devant l'appareil d'une façon précise. Toutes ces interventions ne sont pas sans évoquer les gestes requis pour la préparation d'une lamelle de microscope. Les gros plans de scarifications réalisés par Portman ressemblent ainsi à des lamelles de bactéries. Soigneusement présentées et placées, les «données» du document devaient être compréhensibles une fois traduites sous forme de photographie. L'approche de Portman était étroitement liée aux questions d'observation dont étaient saturées les $N \& Q$. Mais comme David Tomas l'a souligné, «à la différence de ses prédécesseurs qui s'accordaient tous sur ce qu'il fallait chercher, Portman propose une définition de la "photographie scientifique" insistant sur la manière dont on doit voir ce qu'on cherche. " (Tomas 1991: 93) Les clichés de Portman incarnaient une stratégie visuelle destinée à produire un savoir spécifique en supprimant de manière volontaire des éléments excessifs ou perturbateurs.

\section{Les Notes \& Queries}

Les deux textes que je vais maintenant évoquer figurent respectivement dans la troisième (1899) et la quatrième édition (1912) des $N$ \& Q. Ils peuvent être lus ensemble car ils ne sont que très légèrement différents, même si le contexte intellectuel de l'anthropologie a, lui, évolué. Comme l'a monté James Urry, les révisions importantes qui furent apportées à la quatrième édition signalent en effet un changement significatif au niveau des concepts et des méthodes de l'anthropologie, conférant à la discipline une forme manifestement moderne, voire moderniste ${ }^{\mathbf{1 8}}$.

Pourtant, les instructions relatives à la production de documents photographiques restent ambiguës, en particulier dans la quatrième édition, et ce, malgré des références éparses dans différentes sections consacrées aux jeux ou à la danse, par exemple. La troisième édition comporte deux textes sur la photographie: l'un rédigé par Garson, spécialiste d'anthropologie physique, l'autre par Haddon, un zoologue converti à l'anthropologie culturelle ${ }^{19}$. En 1899, la production de documents photographiques suscitait un intérêt grandissant, sans doute parce que les techniques s'avéraient plus accessibles et que la diversité des sujets qu'il devenait possible de photographier ne cessait d'augmenter. Si les $N \& Q$ continuaient de viser un public d'amateurs, le discours conceptuel était destiné aux spécialistes d'une discipline en voie de professionnalisation. Les deux séries d'instructions photographiques reflètent une séparation de plus en plus marquée entre l'anthropologie physique et l'anthropologie culturelle, qui couvait depuis les années 1880 au moins, même si ces deux branches restaient dans une large mesure «théoriquement compatibles» (Kuklick 2009: 56). Les deux textes, en particulier celui de Garson, sont encore largement influencés par une conception hiérarchique des races et des cultures caractéristique du XIX $X^{\mathrm{e}}$ siècle. Mais à la différence des précédentes instructions - celles du racialisme romantique d'im Thurn ou de la taxinomie colonialiste de Portman -, on peut déceler dans les $N \& Q$ l'impact de l'expérience du terrain sur Haddon. Cette nouvelle posture résulte d'un changement de définition du savoir anthropologique: 


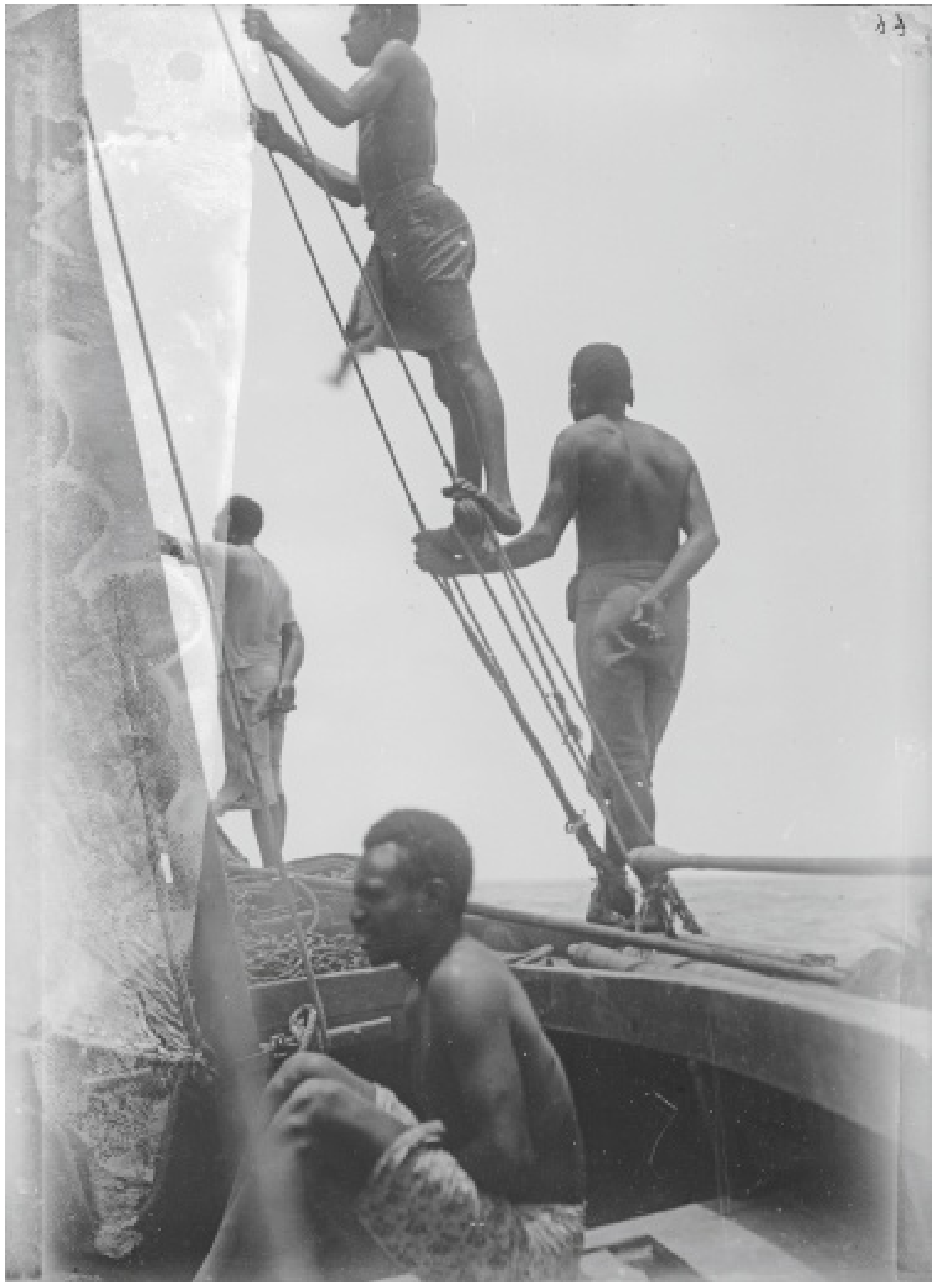

fig. 8

Alfred C. Haddon,

Marins scrutant la mer

à la recherche de dugongs.

Mabuiag Island, Détroit

de Torrès, 1888, épreuve

sur papier albuminé

(C) Cambridge University

Museum of Archaeology

and Anthropology.

N.22795. ACH2. 


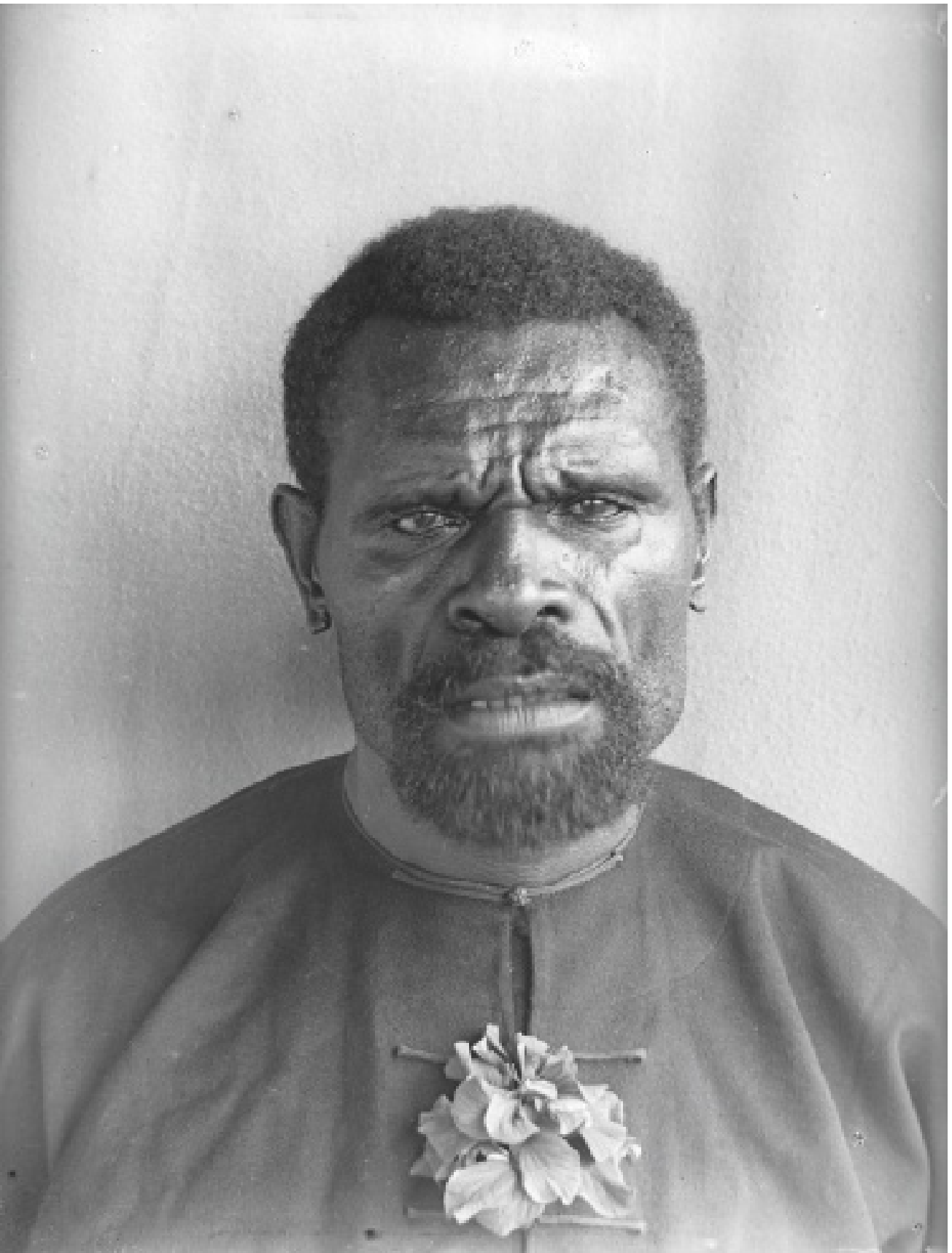

fig. 9

Alfred C. Haddon,

Portrait de Pasi, I'un

des interprètes et ami

de Haddon. Détroit

de Torrès, 1898, épreuve

gélatino-argentique

(C) Cambridge University

Museum of Archaeology

and Anthropology.

N.23108. ACH2. 
celui-ci ne réside plus dans l'ontologie supposée d'un objet offert à la connaissance, comme dans les clichés d'im Thurn et Portman, mais résulte d'une position relationnelle. Les photographies et la production de documents font dès lors partie d'une relation dialogique au terrain. L'abondance de l'expérience elle-même devient le cœur de l'analyse tout en exigeant une transposition scientifique et objective (Hastrup 2004 : 456) [fig. 8].

Haddon s'accordait avec im Thurn sur la difficulté posée par la photographie de «corps vivants»: «On ne doit jamais oublier que quand un indigène pose pour une photographie, il se fige inconsciemment, et on perd dès lors le "jeu" délicat des membres. " (Haddon 1899: 239) Son expérience de terrain dans le détroit de Torrès en 1888-1889 puis en 1898 avait infléchi ses conseils photographiques. II faut souligner ici, pour revenir à la question de l'abondance, que les membres de l'expédition de 1898 avaient emporté une caméra $35 \mathrm{~mm}$ Newman et Guardia et réalisé le premier film anthropologique $^{20}$. [...] Si, dans la pratique, il s'avérait encore difficile d'enregistrer l'«abondance» du terrain, le cadre intellectuel pour le faire existait déjà. La référence fréquente au cinéma dans le travail des anthropologues de l'époque influença leurs approches tant théoriques que pratiques de la photographie.

Pour Haddon, comme pour im Thurn et Portman avant lui, le document possède une forte dimension informative, garantie par le pouvoir mécanique de l'appareil photographique; il ne faut donc pas prendre des photographies «qui ne nous enseignent rien» (Haddon 1899: 238). Sa contribution et celle de Garson offrent néanmoins une approche plus souple qui reconnaît implicitement la place de l'ethnographe dans le désordre du quotidien: «Les actes de la vie de tous les jours " et l'immédiateté requise impliquent de «toujours saisir la première occasion pour prendre des photographies » (ibid. : 240).

Pour Haddon, y compris lors de sa première expédition de 1888 , la photographie participait de la relation aux indigènes rencontrés sur le terrain, quand bien même elle se révélait asymétrique: «La promesse d'un tirage papier permet bien souvent d'obtenir une pose, mais c'est là une faveur et non pas un droit. » (Ibid. : 240) Cette courte phrase indique que Haddon était bien conscient des multiples vies du document, les images utilisées par l'anthropologue étant souvent instantanément considérées comme des portraits à usage privé par les individus photographiés (fig. 9).

En outre, on observe un changement progressif dans l'usage des documents. Haddon voyait dans les habitants des îles du détroit de Torrès des compagnons sympathiques et intelligents. Son travail de terrain l'avait convaincu que ce n'était plus la biologie mais la culture qui était au fondement des étapes du développement humain ${ }^{21}$. Les photographies étaient donc pour lui «des illustrations vivantes» des informations obtenues, dans une création dynamique de savoir, plutôt que des objets d'étude passifs. Cela est notamment visible dans la manière dont il utilisait les photographies. Dans ses publications, par exemple, les images et les légendes sont étroitement liées de manière à créer un document complexe, dont l'autorité et l'authenticité sont garanties par la construction générale de l'ouvrage. Les indigènes y sont eux aussi considérés comme des sources d'autorité et non comme de simples données. Dans les rapports que Haddon consacra à l'expédition dans le détroit de Torrès, publiés sur plus de trente-cinq ans par les presses universitaires de Cambridge, les photographies et le texte renvoient aux individus, dont presque tous les noms sont cités dans les légendes des portraits correspondants (Philp 2004: 93). Cette pratique n'est
20. Les quatre minutes et demie préservées jusqu'à aujourd'hui montrent la fabrication du feu et la célèbre cérémonie de Bomai-Malu, reconstituée pour l'occasion (Griffiths 2002: 131-148). On peut en voir des extraits ici: https://aso.gov.au/ titles/historical/torresstrait-islanders/notes/ (consulté le 5 janvier 2018).

21. Philp 2004 : 93. Jude Philp fait justement remarquer que la quasi-totalité des photographies de terrain prises par Haddon en 1888 montrent davantage l'histoire du détroit de Torrès que son présent. 


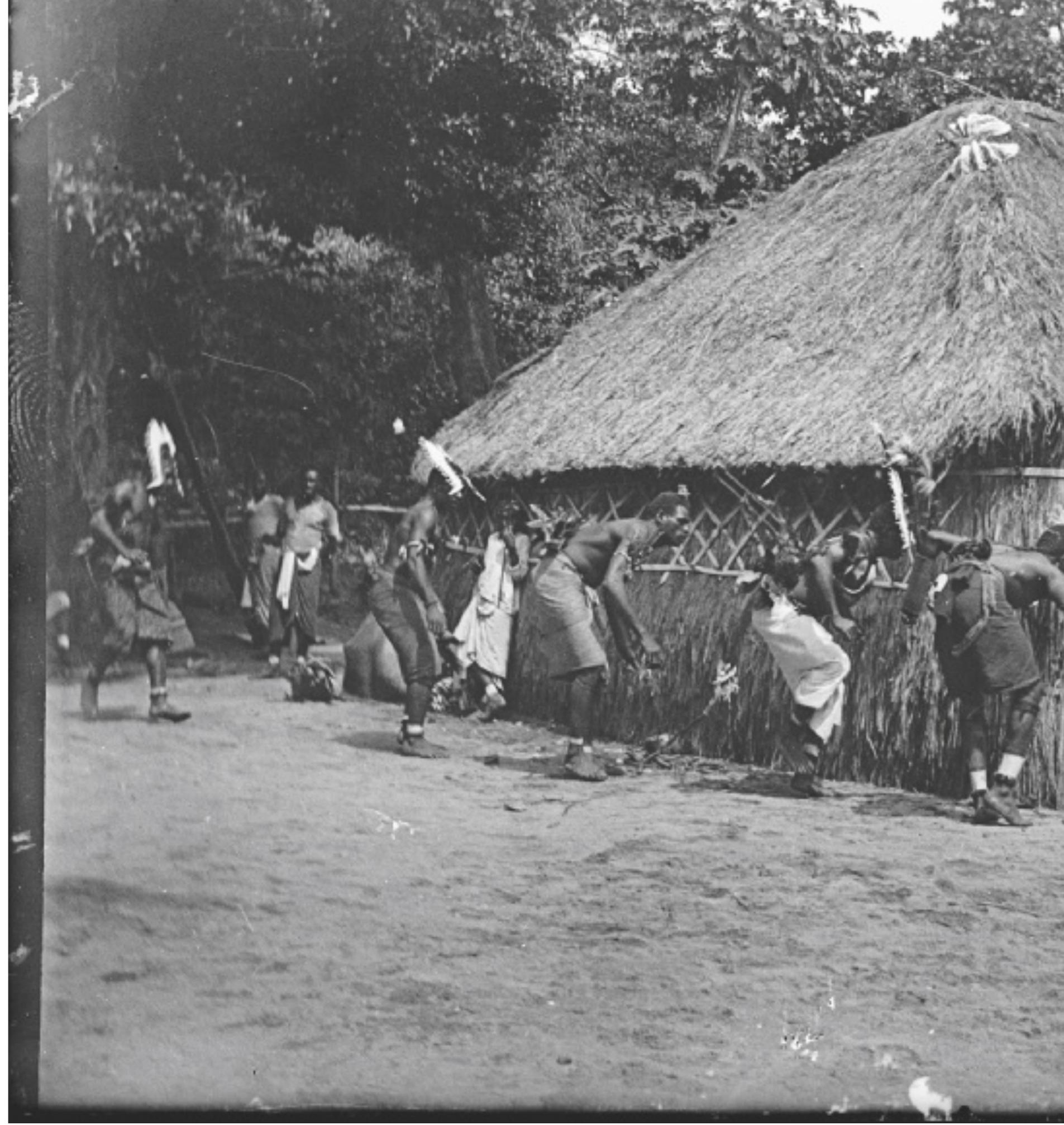


22. Rivers, médecin de formation, s'était tourné vers la psychologie expérimentale. II s'intéressait aux relations entre corps et esprit et on lui doit les premières expériences de psychologie interculturelle, qui ont largement influencé sa méthode anthropologique. Il est surtout connu pour son travail sur les troubles et les traumatismes neurologiques chez les soldats de la Première

Guerre mondiale. Sur Freire-Marreco, qui fut l'une des premières femmes à réaliser une enquête de terrain, voir Blair 2008. pas explicitée ni commentée. Mais j'avancerais l'idée que les instructions de Haddon sur la photographie et la production de documents comme expérience du terrain révèlent les balbutiements d'une nouvelle forme de document visuel en anthropologie.

Cela est particulièrement visible dans la quatrième édition des $N \& Q$. Bien que le texte de Haddon reste globalement inchangé, il y a des différences dans la manière dont certains points sont subtilement mis en avant. $\mathrm{Si}$, dans les textes précédents, en particulier chez Portman, l'observation se concentrait sur la figure individuelle ou sur la technologie, isolées pour les besoins de l'analyse scientifique, le regard anthropologique se déplace de manière presque imperceptible pour embrasser l'excès de la prise de vue: il est désormais «possible de réaliser des vues illustrant différents aspects " (Haddon 1912: 269). C'est le moment où un excès perturbateur se transforme en une abondance productive.

Dans les $N$ \& $Q$ figurent encore des instructions relatives aux photographies de «types» physiques. Mais le langage du document passe de la mathématisation froide de la cartographie somatique, illustrée par Portman ou Garson dans la troisième édition, à une approche plus «naturaliste » et ambivalente: «En plus des profils figés que requiert l'anatomiste, certains portraits devraient être pris de trois quarts ou dans une position conférant une pose plus naturelle et caractéristique. » (Ibid. : 270) À ce titre, il est intéressant de noter que les instructions détaillées de Garson à propos des photographies d'anthropologie physique disparaissent dans la quatrième édition. Après une présentation d'une demi-page, les lecteurs étaient simplement renvoyés au rapport du Comité anthropométrique [Anthropometric Investigation Committee] de la British Association for the Advancement of Science, publié en 1909. De la même manière, la section consacrée à l'anthropologie physique avait été significativement réduite. Le document, y compris celui qui prend le corps pour objet, passait ainsi du biologique au social. Haddon poursuit en ce sens, reprenant un thème introduit pour la première fois dans la troisième édition des $N \& Q$ et qui fait écho au naturalisme physique et culturel d'im Thurn: «Il faut prendre des instantanés de groupes auxquels on n'imposerait aucune pose particulière afin de saisir des attitudes parfaitement naturelles. » (Ibid. : 270) Mais le passage le plus révélateur de ce changement de perspective est le suivant: "Ne négligez pas les gestes du quotidien et faites bien en sorte que le sujet soit réellement en train de réaliser le mouvement; bien des photographies montrant des artisans sont gâchées parce que le sujet a le regard tourné vers le photographe et non vers son travail. » (Ibid. : 271) [fig. 10].

Ce qui modifie véritablement la manière dont on peut lire la production de documents, c'est le contexte qui forme la trame de fond sur laquelle s'est élaborée une nouvelle approche conceptuelle de la photographie. Comme l'ont noté plusieurs commentateurs, la quatrième édition des $N \& Q$ a marqué un changement radical de posture, à la fois théorique et méthodologique. Elle fut éditée par un psychologue distingué, William H. R. Rivers (18641922), dont la méthode généalogique, développée par Haddon lors de l'expédition du détroit de Torrès en 1898, bouleversa la pensée anthropologique. La coéditrice du volume, Barbara Freire-Marreco (1879-1967), avait étudié l'anthropologie à Oxford et réalisé une enquête chez les Pueblos du Sud-Ouest américain ${ }^{22}$. Cette quatrième édition avait toujours pour objectif « de promouvoir une observation anthropologique exacte», mais «tant dans la 
forme que dans le contenu, elle se distingue nécessairement [des éditions précédentes]. Les besoins de l'anthropologie ne sont plus exactement les mêmes; les méthodes utilisées se font plus précises et exigeantes. Et le point de vue, y compris celui de l'observateur amateur, a manifestement changé. » (Read 1912: iv)

Le contenu du volume, dont la rédaction prit cinq ans, «[était] réorganisé selon un schéma scientifique de classification, destiné à montrer les principales divisions de la science et les relations qu'elles entretiennent entre elles.» (Ibid.: iv) La section «Culture» avait été remplacée par un chapitre plus important, intitulé «Sociologie», qui rompait avec les modèles tyloriens du $\mathrm{XIX}^{\mathrm{e}}$ siècle et leurs liens avec l'anthropologie évolutionniste et comparatiste. Le nouveau titre donné à la section consacrée aux questions-clés de l'observation et de la production de documents signale un changement majeur. On peut y voir l'influence des travaux contemporains d'Émile Durkheim comme de la méthode généalogique de Rivers. En outre, les questionnaires avaient été remplacés par des «pages de conseils sur la manière de collecter des données", c'est-à-dire d'observer et de documenter la réalité $\mathbf{2 3}^{2}$. En d'autres termes, le social, dont Haddon avait fait le nouvel objet du document photographique en 1899, émergeait peu à peu comme une entité intégrée qui allait profondément marquer la période classique de l'anthropologie sociale fonctionnaliste britannique après la Première Guerre mondiale. La quatrième édition des $N \& Q$ fut, rappelons-le, utilisée par Bronislaw Malinowski: son expérience de terrain et son texte sur la société des îles Trobriand ont magistralement scellé le destin de la méthode de l'enquête de terrain au sein de l'anthropologie britannique ${ }^{\mathbf{2 4}}$.

\section{Continuités, ambiguïtés, changements}

Tous les textes que nous venons d'évoquer étaient focalisés sur la question de la technique. S'ils varient dans le détail en fonction des préférences personnelles de leurs auteurs et des progrès techniques de l'époque, ils manifestent le même intérêt pour l'outil. En effet, on y trouve de longs passages décrivant les meilleurs appareils, lentilles et produits chimiques. En dépit de certaines différences sur les questions de l'observation et des modes de présentation des faits anthropologiques, tous évoquent ces multiples aspects techniques, même si, comme le rappelait Portman, ceux qui prennent des photographies «préféreraient sans doute consacrer leur temps à enregistrer des faits anthropologiques plutôt qu'aux opérations photographiques » (Portman 1896: 80). Ces précisions sur le matériel à utiliser ont pourtant été largement ignorées par les commentateurs, qui n'ont lu ces textes qu'en fonction des modes de prises de vue et des codes de représentation à adopter, ou qui n'ont vu dans la technique photographique qu'une métaphore grossière de la relation coloniale, incarnée par exemple dans le rapport entre longueur de focale et distance culturelle, ou encore dans les implications fonctionnalistes du grand-angle 25 (Mclntyre et MacKenzie 1992; Young 1999: 19-20).

En apparence, donc, ces passages techniques semblent seulement indiquer quel appareil utiliser et quel type de plaques ou de produits chimiques privilégier dans les climats tropicaux ou, à l'inverse, secs et chauds. II me semble cependant que, comme on le sait pour d'autres branches de l'histoire des sciences, cette attention portée aux instruments
23. À propos de ces influences sur les $N \& Q$, voir Urry 1972: 51. Sur l'anthropologie britannique en général et l'influence de Rivers, voir Kuklick 2009.

24. Voir Kuklick 2009: 65. Cette lecture relève à bien des égards d'une sorte de "mythe fondateur". En effet, les méthodes auxquelles Malinowski eut recours sur le terrain avaient déjà été en partie utilisées par Haddon, Rivers, Baldwin Spencer ou encore Diamond Jenness.

25. Certains auteurs tissent des liens directs entre le style de la pratique photographique des anthropologues et leur ancrage épistémologique. Pour Michael Young, par exemple, les photographies de Malinowski reflètent ainsi sa méthodologie fonctionnaliste naissante. $(\mathrm{NdT})$ 
- appareils, plaques de verre, films, produits chimiques, bacs, châssis, chambres noires et toiles de fond - constitue un paramètre discursif permettant de garantir la validité, la lisibilité et la fonction anthropologique de documents photographiques «montrant les choses d'une certaine manière » (Portman 1896: 80). Comme le précise Portman dans un passage sur les verres de mise au point, «[son] objet est d'obtenir un grand nombre de détails précis » (ibid. : 81).

Cette focalisation sur la production de documents photographiques servait à imposer une certaine perception du document visuel dans une époque d'incertitude. Grimshaw rappelle ainsi que «rien n'angoissait plus les figures majeures de l'anthropologie du xx siècle en Grande-Bretagne que le spectre du gentleman amateur, ébloui par l'instrumentation scientifique, qui collectionne et classe dans une optique muséale» (Grimshaw 2001: 3). La nouvelle anthropologie avait délaissé tous les outils qui, telles des prothèses, servaient à collecter des données. Elle privilégiait désormais le corps observant de l'anthropologue sur le terrain. Le photographe devenait lui aussi un «document » qui témoignait de son implication et de sa légitimité: il valorisait les pratiques de l'observation directe et bien renseignée et, dans le même temps, il «contenait en lui la menace de la communication, de l'échange et de la présence - autant de facteurs remettant en cause la prétention à l'objectivité de l'ethnographe» (Poole 2005 : 166).

De manière significative, l'émergence de la nouvelle anthropologie du $x x^{e}$ siècle coïncide, de fait, avec la marginalisation, voire la disparition, des techniques utilisées jusque-là: la création de documents visuels avait fini par être assimilée au corps de l'ethnographe. Comme le faisait remarquer Rivers, l'anthropologue, seul sur son terrain, « devenait un instrument scientifique incarné» (Kuklick 2009: 65). La constitution d'un savoir et la création de documents ont bien sûr toujours reposé sur la présence d'un corps observant et sur l'instantanéité du témoignage ou du récit d'un individu présent "sur place», garant de l'exactitude. Mais l'anthropologie évoluait de plus en plus vers un état d'immersion culturelle et d'intention objective que l'ethnographe avait simultanément incorporées; là, l'excès observé se transformait en une expérience de l'abondance. En outre, l'acte photographique lui-même et les relations entre l'ethnographe, l'appareil photographique et le sujet photographié constituaient un réseau d'humains et de non-humains, au sens latourien, permettant au document d'émerger. Les commentaires sur les indigènes se détournant de l'appareil, ce qu'im Thurn trouvait intéressant d'un point de vue anthropologique mais évidemment frustrant pour le photographe, révèlent que les anthropologues ne cessaient de s'interroger sur la façon de saisir les comportements culturels (im Thurn 1893: 188). De même, les descriptions très détaillées de Portman sur l'intersubjectivité des sujets posant, ou les conseils de Haddon sur la mise au point et l'usage de miroirs orientés vers la lentille pour réaliser des photographies non intrusives, renforcent cette mise en relation du photographe, de l'appareil et du sujet photographié (Portman 1896: 76-77; Haddon 1899: 236). Les transformations qui affectèrent cette relation triangulaire témoignent de changements progressifs mais notables, concernant aussi bien l'épistémologie que le document. À l'opposé d'une instrumentation externe et prothétique, le corps de l'ethnographe devenait ce nouveau lieu incarné de l'observation 
où se rejoignaient la subjectivité personnelle en jeu dans l'observation et la distance impliquée par le geste d'observer. [...]

Les passages restés à l'identique entre la troisième et la quatrième édition des $N \& Q$ indiquent très précisément des changements de valeurs au sein de la discipline. Les documents photographiques donnent une forme concrète à l'illusion de la représentation anthropologique: «[...] Vous êtes là... parce que j'étais là. » (Clifford 1996: 30) Les photographies revêtent dès lors une force métaphorique et deviennent le moyen privilégié de communiquer au lecteur un sentiment d'immersion culturelle, forme de substitut à l'expérience personnelle du terrain; elles garantissent ce qui aurait pu être vu par le lecteur si, lui aussi, il avait été là. Mais pris dans le désordre et la contingence de la rencontre, l'ethnographe doit rester invisible s'il veut valider scientifiquement ses observations et ses photographies. C'est précisément pour cette raison que le passage sur les paramètres de l'image cité plus haut est si important. La photographie n'a pas seulement pour mission d'enregistrer, elle doit aussi préserver l'autorité de l'ethnographe et l'illusion de la représentation. À travers une confusion de plus en plus grande entre un naturalisme photographique et un naturalisme de l'observation (Tucker 2005: 7), ces documents participent aussi du mécanisme propre à la discipline, qui consiste à transformer des méthodes de collecte extrêmement personnelles, inscrites dans le temps et intersubjectives, en des preuves convaincantes (Engelke 2008: S2).

Soulignons enfin que, à cette époque, les documents photographiques cessèrent d'être des ressources centralisées pour la diffusion des idées anthropologiques et devinrent des documents privés ramenés du terrain. Les photographies ne circulaient plus à travers différents réseaux, elles n'étaient plus rassemblées dans des archives d'images et de diapositives pour lanterne magique, comme à l'époque précédente. Elles étaient réunies autour de l'ethnographe, désormais centre incarné du savoir anthropologique. Quand Malinowski, qui avait emporté avec lui la quatrième édition des $N \& Q$, revint des îles Trobriand, il n'archiva pas ses clichés. Ceux-ci n'étaient qu'un produit de son terrain parmi d'autres et lui servirent à analyser son matériau, c'est-à-dire à gérer l'abondance de son expérience: «En mettant en forme mon matériau sur les jardins, je constate que le fait de contrôler mes notes de terrain par des photographies m'amène à reformuler un très grand nombre de points ${ }^{26}$. (Malinowski 1932: 461) Alors que le corps de l'ethnographe devenait le lieu de l'observation, la photographie n'était plus une médiation de l'expérience scientifique mais une extension, une prothèse de la personne de l'ethnographe sur le terrain, «à la fois objet ethnographique, catégorie analytique et orientation méthodologique " (Riles 2006: 7). $[\ldots]$
26. Edward EvansPritchard, qui travailla au Soudan dans les années 1920 et 1930 , déposa ses archives visuelles au Pitt Rivers Museum de l'université d'Oxford dans les années 1960 après avoir fini de les exploiter. On retrouve le même procédé chez un anthropologue spécialiste de l'Afrique du Sud, Isaac Shapera, qui fit don de ses photographies au Royal Anthropological Institute. 


\section{Alvarez Roldán, Arturo}

1992 « Looking at Anthropology from a Biological Point of View: A. C. Haddon's Metaphors on Anthropology", History of the Human Sciences 5 (4): 21-32.

\section{Banks, Marcus et Ruby, Jay}

2011 «Introduction: Made to Be Seen ", in Marcus Banks et Jay Ruby (dir.), Made to Be Seen: Historical Perspectives in Visual Anthropology. Chicago, University of Chicago Press: 1-18.

\section{Blair, Mary Ellen}

2008 A Life Well Led: The Biography of Barbara Freire-Marreco Aitken, British Anthropologist. Santa Fe, Sunstone Press.

\section{British Association for the Advancement of Science}

1874 Notes and Queries on Anthropology. Londres, Edward Stanford.

\section{Clifford, James}

1996 [1988] Malaise dans la culture: I'ethnologie, la littérature et l'art au XX' siècle, trad. de I'anglais par Marie-Anne Sichère. Paris, École nationale supérieure des beaux-arts.

\section{Cox, Amy}

2007 «Purifying Bodies, Translating Race: The Lantern Slides of Sir Everard im Thurn ", History of Photography 31 (4): 348-364.

\section{Daston, Lorrain et Galison, Peter}

1992 "Images of Objectivity", Representations 40 : 81-128.

\section{Dalziell, Rosamund}

2007 "Everard im Thurn in British Guiana and the Western Pacific", in Peter Hulme et Russell McDougall (dir.), Writing, Travel and Empire. Londres, I.B. Tauris: 97-117.

\section{Dias, Nélia}

1994 «Photographier et mesurer: les portraits anthropologiques ", Romantisme 84 : 37-49.

\section{Edwards, Elizabeth}

1990 "Photographic "Types" The Pursuit of Method ", Visual Anthropology 3 (2-3) : 239-258.

1992 "Visualising Science», in Elizabeth Edwards (dir.), Anthropology and Photography, 1860-1920. New Haven / Londres, Yale University Press / Royal Anthropological Institute: 108-121.

2001 Raw Histories: Photographs, Anthropology and Museums. Oxford / New York, Berg.

\section{Engelke, Matthew}

2008 "The Objects of Evidence", Journal of the Royal Anthropological Institute 14 (1), supplément S1: 1-21.

\section{Griffiths, Alison}

2002 Wondrous Difference: Cinema, Anthropology, and Turn-of-the-Century Visual Culture. New York, Columbia University Press.

\section{Grimshaw, Anna}

2001 The Ethnographer's Eye. Cambridge, Cambridge University Press.

\section{Haddon, Alfred Cort}

1897 "The Saving of Vanishing Knowledge», Nature 55: 305-306.

1899 "Photography", in Notes and Queries on Anthropology, $3^{e}$ édition. Londres, Royal Anthropological Institute : 240-246

1910 History of Anthropology. Londres, Watts \& Co.

1912 "Photography", in Notes and Queries on Anthropology, $4^{\mathrm{e}}$ édition. Londres, Royal Anthropological Institute: 267-271

\section{Hastrup, Kirsten}

2004 "Getting It Right: Knowledge and Evidence in Anthropology ", Anthropological Theory 4 (4) : 455-472.

\section{Im Thurn, Everard}

1893 «Anthropological Uses of the Camera ", Journal of the Anthropological Institute 22 : 184-203

\section{Kuklick, Henrietta}

1991 The Savage Within:

The Social History of British Social Anthropology 1885-1945.

Cambridge, Cambridge University Press.

2009 "The British Tradition», in Henrietta Kuklick (dir.), A New History of Anthropology. Oxford, Blackwell : 52-78.

\section{Lamprey, John}

1869 «On a Method of Measuring the Human Form ", Journal of the Ethnological Society 1 (1) : 84-85.

\section{Malinowski, Bronislaw}

1932 Coral Gardens and Their Magic. Londres, Routledge.

\section{Mclntyre, Martha et MacKenzie, Maureen}

1992 "Focal Length as an Analogue of Cultural Distance ", in Elizabeth

Edwards (dir.), Anthropology and Photography, 1860-1920.

New Haven / Londres, Yale

University Press / Royal

Anthropological Institute: 158-163.

\section{Neumayer, Georg}

1875 Anleitung zu

wissenschaftlichen Beobachtungen auf Reisen. Berlin,

Robert Oppenheim Verlag.

\section{Philp, Jude}

2004 " "Embryonic Science": The 1888 Torres Strait Photographic Collection of A. C. Haddon", in Richard Davies (dir.), Woven Histories, Dancing Lives: Torres Strait Islander Identity, Culture and History. Canberra, Aboriginal Studies Press for the Australian Institute of Aboriginal and Torres Strait Islander Studies: 90-106.

\section{Pinney, Christopher}

1992 «The Parallel Histories of Anthropology and Photography ", in Elizabeth Edwards (dir.), Anthropology and Photography, 1860-1920. New Haven / Londres, Yale University Press / Royal Anthropological Institute: 74-92.

1997 Camera Indica. Londres, Reaktion.

2003 "Introduction ", in Christopher Pinney et Nicolas Peterson (dir.) Photography's Other Histories. Durham, Duke University Press : 2-16.
Read, Charles Hercules

1912 "Preface", in Notes and

Queries on Anthropology, $4^{\mathrm{e}}$ édition. Londres, Royal Anthropological Institute: III-VII.

\section{Riles, Annelise}

2006 "Introduction: In response», in Documents: Artifacts of Modern Knowledge. Ann Arbor, University of Michigan Press : 2-38.

\section{Sen, Satadru}

2009 «Savage Bodies, Civilized Pleasures: M. V. Portman and the Andamanese ", American Ethnologist 36 (2) : 364-379.

\section{Sera-Shriar, Efram}

2013 The Making of British Anthropology, 1813-1871. Londres, Pickering and Chatto.

\section{Stocking, George W. Jr.}

1995 After Tylor: British Social Anthropology 1888-1951. Londres, Athlone Press. 


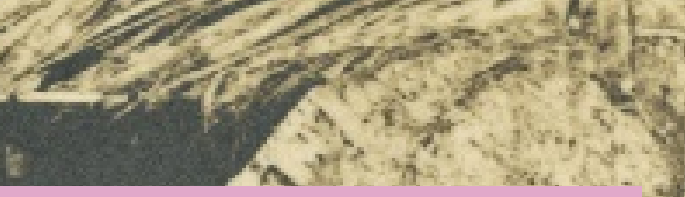

\section{Tayler, Donald}

1992 «Very Lovable Human Beings:

The Photography of Everard

im Thurn ", in Elizabeth Edwards (dir.), Anthropology and Photography, 1860-1920. New Haven / Londres,

Yale University Press / Royal

Anthropological Institute: 187-192.

\section{Tomas, David}

1991 «Tools of the Trade:

The Production of Ethnographic

Observation in the Andaman Islands 1858-1922 ", in George W. Stocking Jr. (dir.), Colonial Situations. Madison, University of Wisconsin Press: 75-108.

\section{Tucker, Jennifer}

2005 Nature Exposed: Photography as Eyewitness in Victorian Science.

Baltimore, Johns Hopkins University Press.

\section{Urry, James}

1972 "Notes and Queries on

Anthropology and the Development of Field Methods in British Anthropology, 1870-1920, Proceedings of the Royal Anthropological Institute 1972 45-57.

\section{Young, Michael}

1999 Malinowski's Kiriwina:

Fieldwork Photography 1915-1918.

Chicago, University of Chicago

Press.

\section{Zimmerman, Andrew}

2001 Anthropology and Antihumanism in Imperial Germany. Chicago, University of Chicago Press.

page $\mathbf{3 0}$ et ci-contre Everard im Thurn, Jeunes hommes Warrau jouant, Guyane britannique, vers 1883-1884 (détail), épreuve sur papier albuminé. Roya Anthropological Institute, Londres (c) RAI 641

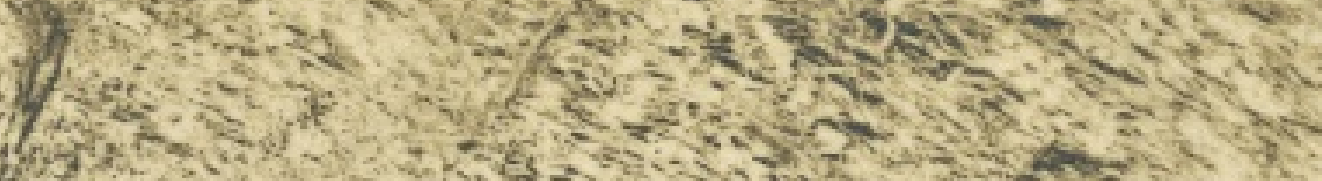

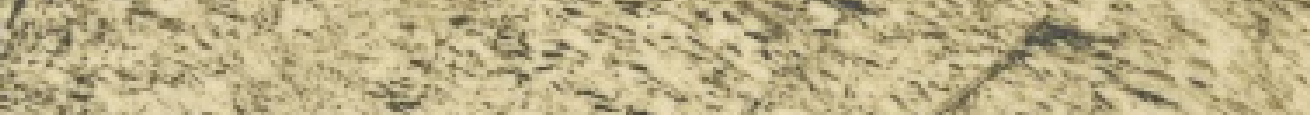

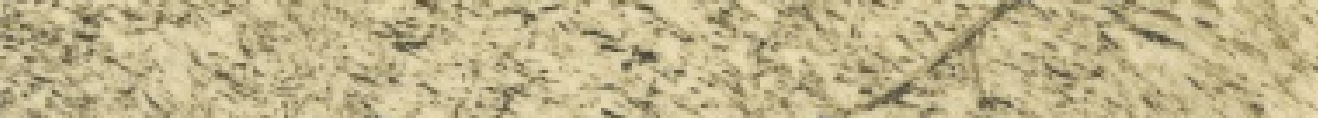

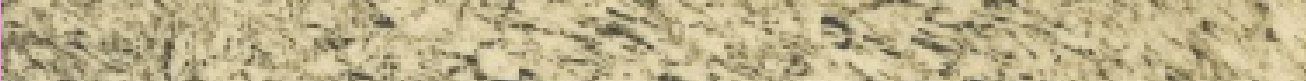

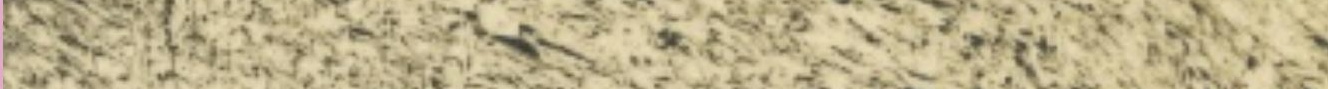

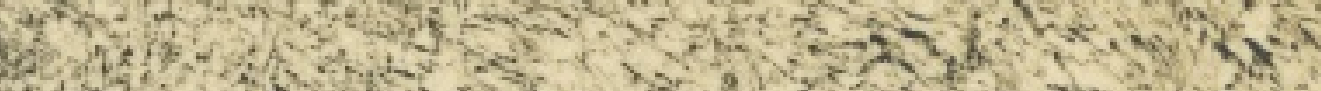

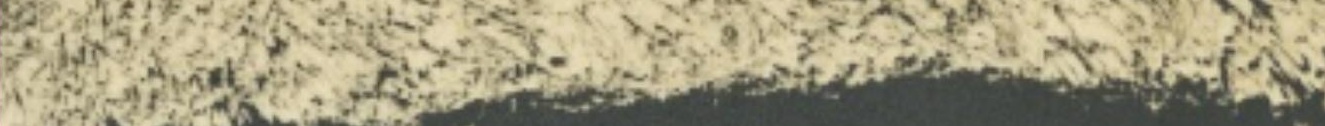

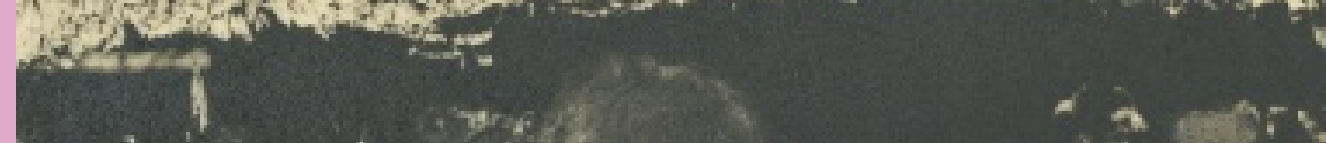
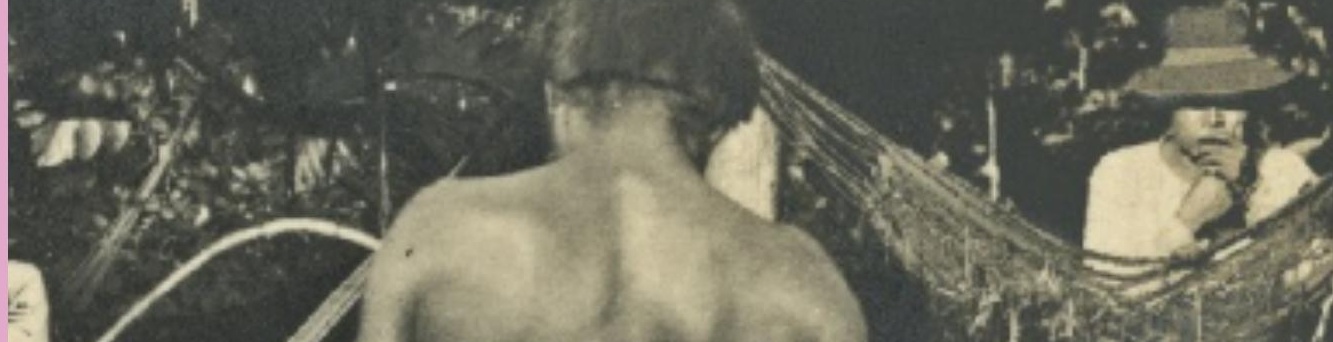

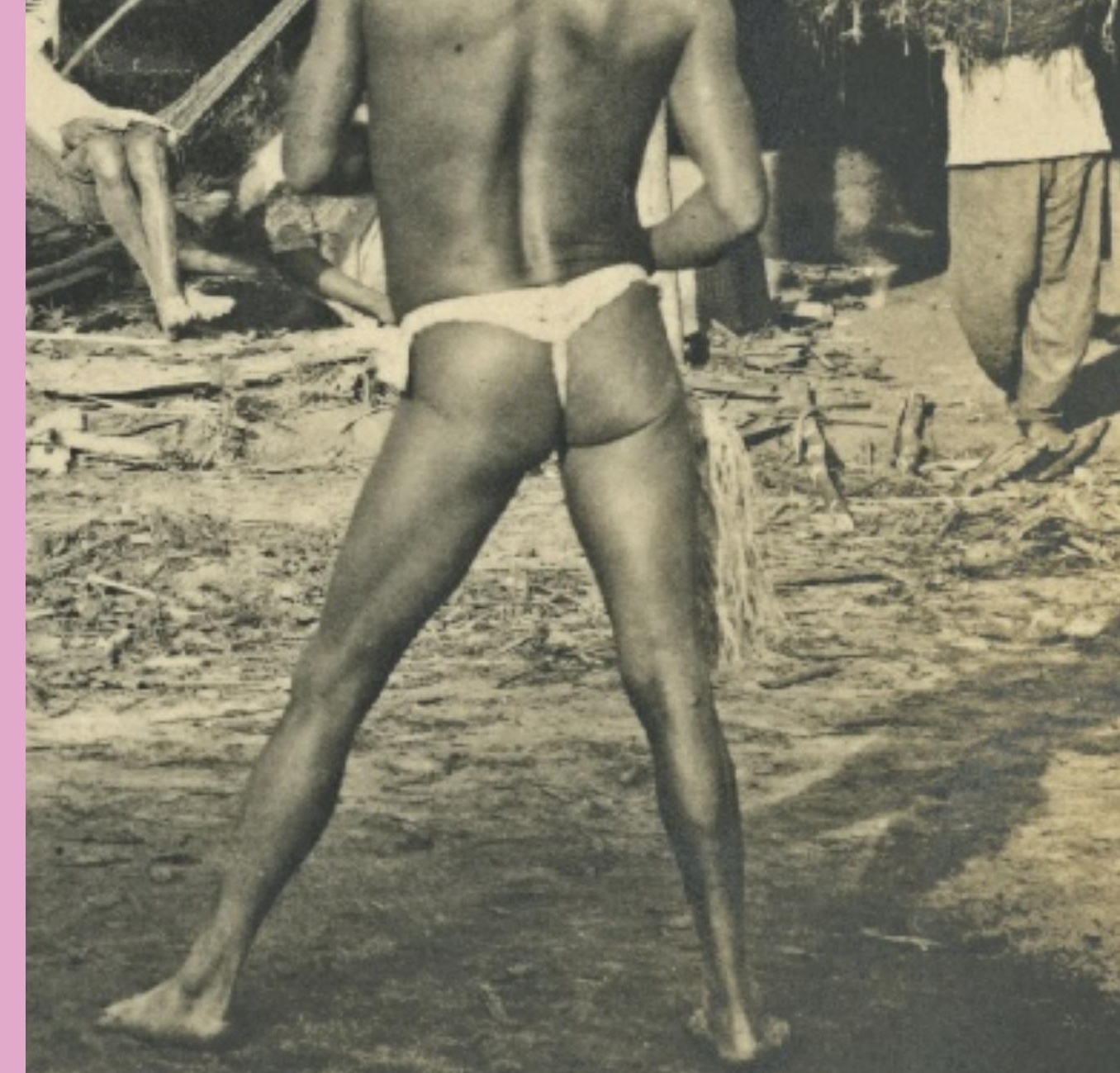

